\title{
Small-scale lithospheric heterogeneity characterization using Bayesian inference
}

Itahisa González Álvarez¹ (eeinga@leeds.ac.uk, @seismowaves)

Sebastian Rost ${ }^{1}$ (s.rost@leeds.ac.uk)

Andy Nowacki ${ }^{1}$ (a.nowacki@leeds.ac.uk)

Neil D. Selby² (neil@blacknest.gov.uk)

${ }^{1}$ School of Earth and Environment, University of Leeds, Leeds, LS2 9JT, UK.

${ }^{2}$ Blacknest, AWE, Reading, RG7 4RS, UK

This manuscript has been submitted for publication in Geophysical Journal International and is undergoing peer-review. Please note that the manuscript has not been formally accepted for publication. Subsequent versions of this manuscript may have slightly different content. If accepted, the final version of this manuscript will be available via the Peer-review Publication DOI link on this webpage. Please feel free to contact the authors; We welcome feedback. 


\title{
Small-scale lithospheric heterogeneity characterization using Bayesian inference.
}

\author{
Itahisa González Álvarez ${ }^{1}$, Sebastian Rost ${ }^{1}$, Andy Nowacki ${ }^{1}$, \\ and Neil D. Selby ${ }^{2}$ \\ $1_{\text {School of Earth and Environment, University of Leeds, LS2 9JT, UK. E-mail: eeinga@leeds.ac.uk }}$ \\ ${ }^{2}$ Blacknest, AWE, Reading, RG7 4RS, UK
}

\begin{abstract}
SUMMARY
Observations from different disciplines have shown that our planet is highly heterogeneous at multiple scale lengths. Still, many seismological Earth models tend not to include any small-scale heterogeneity or lateral velocity variations, which can affect measurements and predictions based on these homogeneous models. In this study, we describe the lithospheric small-scale heterogeneity structure in terms of the intrinsic, diffusion and scattering quality factors, as well as an autocorrelation function, associated with a characteristic scale length $(a)$ and root mean square (RMS) fractional velocity fluctuations $(\epsilon)$. To obtain this characterization, we combined a single-layer and a multi-layer energy flux models with a new Bayesian inference algorithm. Our synthetic tests show that this technique can successfully retrieve the input parameter values for 1- or 2-layer models and that our Bayesian algorithm can resolve whether the data can be fitted by a single set of parameters or a range of models is required instead, even for very complex posterior probability distributions. We applied this technique to three seismic arrays in Australia: Alice Springs array (ASAR), Warramunga Array (WRA) and Pilbara Seismic Array (PSA). Our single-layer model results suggest intrinsic and diffusion attenuation are strongest for ASAR, while scattering and total attenuation are similarly strong for ASAR and WRA. All quality factors take higher values for PSA than for the other two arrays, implying that the structure beneath this array is less attenuating and heterogeneous than for ASAR or WRA. The multi-layer model results shows the crust is more heterogeneous than the lithospheric mantle for all
\end{abstract}


arrays. Crustal correlation lengths and RMS velocity fluctuations for these arrays range from $\sim 0.2-1.5 \mathrm{~km}$ and $\sim 2.3-3.9 \%$ respectively. Parameter values for the upper mantle are not unique. Both low $(<2$ $\mathrm{km})$ and high $(>5 \mathrm{~km})$ correlation length values are equally likely and $\epsilon$ takes values up to $\sim 6 \%$ and $\sim 7 \%$ for ASAR and WRA respectively and up to $\sim 3 \%$ for PSA. We attribute the similarities in the attenuation and heterogeneity structure beneath ASAR and WRA to their location on the proterozoic North Australian Craton, as opposed to PSA, which lies on the archaean West Australian Craton. Differences in the small-scale structure beneath ASAR and WRA can be ascribed to the different tectonic histories of these two regions of the same craton. Overall, our results highlight the suitability of this technique for future scattering and small-scale heterogeneity studies, since our approach allows us to obtain and compare the different quality factors, while also giving us detailed information about the trade-offs and uncertainties in the determination of the scattering parameters.

Keywords: Structure of the Earth, Australia, statistical methods, coda waves, seismic attenuation, wave scattering and diffraction. 


\section{INTRODUCTION}

2 The Earth is heterogeneous on a variety of scales, ranging from the grain scale

to scales of hundreds of kilometers. This heterogeneity is evident in data from geo-disciplines with varying sensitivity to scales, such as geochemistry, mineralogy or seismology (e.g. Wu and Aki, 1988). Due to the seismic wavelengths, most seismological Earth models are laterally homogeneous or smoothly varying, with a lack of small-scale heterogeneity (e.g. Helmberger, 1968; Dziewonski and Anderson, 1981; Kennett and Engdahl, 1991; Randall, 1994). This limits our understanding of high-frequency seismic wave propagation and challenges in seismic imaging of small-scale heterogeneities remain.

Many seismic studies published before the 1970s were based on laterally homogeneous Earth models (e.g. Alexander and Phinney, 1966) which were able to explain the propagation of long period signals, but failed to explain high frequency seismograms. Aki (1969) showed that the power spectra of coda waves for a given station are independent of epicentral distance and earthquake magnitude. He proposed that codas were caused by backscattered energy from discrete heterogeneities randomly distributed beneath the stations. The presence and shape of the coda strongly depends on the heterogeneity structure and the geology beneath the station. Later studies (e.g. Aki and Chouet, 1975; Rautian and Khalturin, 1978) showed that the stable decay in coda wave amplitude was also independent of epicentral distance and source mechanism, fully supporting the scattering hypothesis.

Methods to study heterogeneity and scattering within the Earth vary depending on the type of the heterogeneity. Many seismological studies use deterministic methods to characterize the structure of the Earth (e.g. Christensen and Mooney, 1995; Zelt and Barton, 1998) or to find individual scatterers and try to obtain their 
particular characteristics and locations (e.g. Etgen et al., 2009). Marchenko imaging (e.g. Thorbecke et al., 2017; van der Neut et al., 2015) or migration techniques (e.g. Etgen et al., 2009) are often used in reflection seismology to study shallow structure and are a good example of deterministic methods. These techniques tend to have limited spatial resolution due to the wavelength of the studied waves and do not take into account small-scale heterogeneities (on the order of magnitude of the wavelength), therefore failing to explain or reproduce the complex coda waves we see in seismograms. Therefore, a stochastic description of the heterogeneity distribution is often necessary for scattering studies (e.g. Korn, 1990, 1997; Margerin, 2005; Hock et al., 2004; Ritter et al., 1998).

A stochastic approach (e.g. Frankel and Wennerberg, 1987; Shapiro and Kneib, 1993; Hock et al., 2004) gives a statistical description of the structure and determines the integrated effect of heterogeneity on seismic waves propagating through it, so the characteristics and locations of individual scatterers are not relevant. Studies (e.g. Aki, 1973; Flatté and Wu, 1988; Langston, 1989) showed the crust and lithospheric heterogeneity to be statistically complex and the necessity of heterogeneous Earth models that were capable of explaining not only the main waveforms but also coda waves. Seismic wave propagation through heterogeneous stochastic media can be described using several methods. Single-scattering perturbation theory (e.g. Aki and Chouet, 1975; Sato, 1977, 1984) considers scattering to be a weak process and coda waves the superposition of single scattered waves generated at randomly distributed heterogeneities within the Earth. It often makes use of the Born approximation (e.g. Sato et al., 2012), a first-order perturbation condition which does not take into account the energy loss from the primary waves. As a result, energy is not conserved in the scattering process (e.g. Aki and Chouet, 1975). Radiative transfer theory, initially developed for light propagation (Chan- 
drasekhar, 1950) and later modified for and applied to seismology, has been used in several scattering and attenuation studies (e.g. Margerin, 2005; Sato et al., 2012; Wu, 1985; Fehler et al., 1992).

In this study, we combine two stochastic methods, the single layer modified Energy Flux Model (EFM, Korn, 1990) and the depth dependent Energy Flux Model (EFMD, Korn, 1997), with a Bayesian inversion algorithm which allows us to characterise small-scale lithospheric heterogeneity by fully exploring the scattering parameter space and obtain information about the trade offs and uncertainties in the determination of the parameters. We applied these methods to a large dataset of teleseismic events recorded at three seismic arrays of the Australian National Seismic Network: Alice Springs Array (ASAR) and Warramunga Array (WRA), which are primary seismic arrays from the International Monitoring System network, and Pilbara Seismic Array (PSA). 


\section{METHODS}

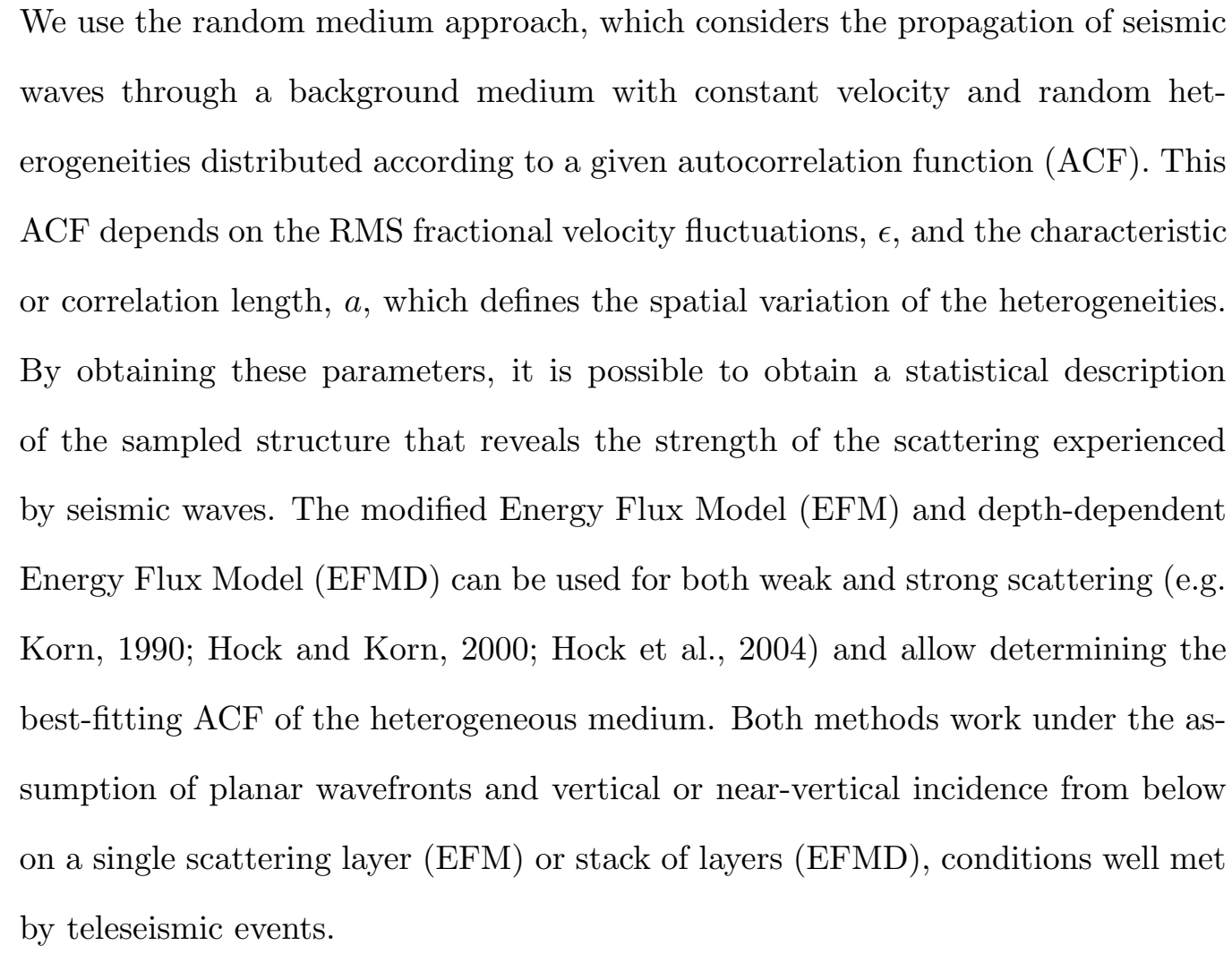
waves through a background medium with constant velocity and random heterogeneities distributed according to a given autocorrelation function (ACF). This $\mathrm{ACF}$ depends on the RMS fractional velocity fluctuations, $\epsilon$, and the characteristic or correlation length, $a$, which defines the spatial variation of the heterogeneities. By obtaining these parameters, it is possible to obtain a statistical description of the sampled structure that reveals the strength of the scattering experienced by seismic waves. The modified Energy Flux Model (EFM) and depth-dependent Energy Flux Model (EFMD) can be used for both weak and strong scattering (e.g. Korn, 1990; Hock and Korn, 2000; Hock et al., 2004) and allow determining the best-fitting ACF of the heterogeneous medium. Both methods work under the assumption of planar wavefronts and vertical or near-vertical incidence from below on a single scattering layer (EFM) or stack of layers (EFMD), conditions well met by teleseismic events.

Here we present a short introduction to the EFM and EFMD. Full details about the methods can be found in Korn (1990), Korn (1997), Hock and Korn (2000) and Hock et al. (2004).

\subsection{The Modified Energy Flux Model for a single scat- tering layer}

When a plane wavefront enters a heterogeneous unlayered medium from below, part of the energy propagates with the ballistic wavefront, while part forms the forward scattered coda energy that arrives later at the surface and some energy scatters back into the half-space. Total energy $E_{t o t}$ is conserved in this process 
90 and we can write it in terms of frequency, $\omega$, and time, $t$, as

$$
E_{t o t}(\omega, t)=E_{d}(\omega, t)+E_{c}(\omega, t)+E_{\text {diff }}(\omega, t),
$$

with $E_{d}$ being the energy of the direct wave, $E_{c}$ the energy transferred from the direct wave into the coda (forward scattered) and $E_{\text {diff }}$ the energy diffusion (backscattering) from the current layer back into the half-space. The energy that is transferred from the incoming wavefront to the scattered coda and the backscattering to the half-space can be expressed as an energy loss for the direct wave, controlled by a quality factor $Q_{s}$ for scattering and $Q_{\text {diff }}$ for diffusion. To take into account anelastic (intrinsic) attenuation, we use the quality factor $Q_{i}$. The EFM assumes spatially homogeneous coda energy within the scattering layer. Energy transfer into the coda due to scattering or anelastic losses stops once the ballistic wave leaves the scattering layer after totally reflecting at the free surface, while diffusion out of the scattering layer can continue after that.

A linear least-squares fit of the theoretical coda power spectral density allows us to calculate the coda decay rate, $a_{1}$, and its amplitude at zero time, $a_{0}$ (Korn, 1990, 1993). The values of $Q_{i}$ and $Q_{d i f f}$ at $1 \mathrm{~Hz}, Q_{i 0}$ and $Q_{d 0}$, can be obtained from values of $a_{1}$ at different frequencies via

$$
a_{1}(\omega)=-2 \pi\left[Q_{d 0}^{-1}+Q_{i 0}^{-1}(\omega / 2 \pi)^{1-\alpha}\right] \log _{10} e,
$$

where $\alpha$ is the exponent controlling the frequency dependence of $Q_{i}$ (Korn, 1990, Eq. 17). Eqs. 16 and 17 from Korn (1990) allow us to determine $Q_{\text {diff }}$ and $Q_{i}$ at different frequency bands. Laboratory measurements of $\alpha$ have shown that it probably remains below 1 for most of the frequency range considered here (Korn, 1990, and references therein). Our attempts at obtaining $\alpha$ as a third free param- 
eter in the least-squares inversion of Eq. 2 revealed a very complicated trade-off with $Q_{i 0}$ and $Q_{d 0}$, with high values of $\alpha$ corresponding to negative values of $Q_{i 0}$ and/or $Q_{d 0}$. Therefore, we limited $\alpha$ to the range of $0.0-0.6$, in steps of 0.1 , and chose the value that minimised the misfit to the data. Both our results and those of Korn (1990) show that $\alpha$ has a strong effect on $Q_{i}$ but only weakly affects $Q_{\text {diff }}$. The impossibility to fully invert for $\alpha$ makes it difficult to accurately calculate $Q_{i}$ with the EFM. However, given that $Q_{i}$ is generally much larger than $Q_{\text {diff }}$, since diffusion becomes more important with increasing source distance (Korn, 1990), we can expect the effect of anelasticity on coda levels to be small.

The coda amplitude at zero time, $a_{0}$, is related to $Q_{s}$ through

$$
Q_{s} \approx 2 I_{D} \omega 10^{-a_{0}},
$$

$I_{D}$ being the integral of the squared amplitude envelope, $A^{2}(t ; \omega)$, over the time window of the direct wave arrival (Hock and Korn, 2000). We can then use the relationships between $Q_{s}^{-1}$ and the structural parameters for different types of ACFs obtained by Fang and Müller (1996) to determine the type of ACF that fits the data best, as well as a first estimation of the correlation length $(a)$ and the RMS velocity fluctuations $(\epsilon)$ for a single scattering layer.

Finally, the total quality factor, $Q_{t o t}$, can be calculated as:

$$
\frac{1}{Q_{t o t}}=\frac{1}{Q_{\text {diff }}}+\frac{1}{Q_{i}}+\frac{1}{Q_{s}}
$$

The eight different one octave-wide frequency bands we used in our analysis for both methods are shown in Table 1. 
Table 1: List of all frequency bands used in this study.

\begin{tabular}{ccccccccc}
\hline Frequency band & $\mathrm{A}$ & $\mathrm{B}$ & $\mathrm{C}$ & $\mathrm{D}$ & $\mathrm{E}$ & $\mathrm{F}$ & $\mathrm{G}$ & $\mathrm{H}$ \\
\hline Minimum frequency $(\mathrm{Hz})$ & 0.5 & 0.75 & 1 & 1.5 & 2 & 2.5 & 3 & 3.5 \\
\hline Maximum frequency $(\mathrm{Hz})$ & 1.0 & 1.5 & 2 & 3 & 4 & 5 & 6 & 7 \\
\hline
\end{tabular}

\subsection{The Energy Flux Model for depth-dependent het- erogeneity}

Korn (1997) modified the EFM to include depth-dependent heterogeneity. In this model, a plane wavefront enters a stack of $N$ heterogeneous layers from below. Each layer $j$ has its own characteristic transit time $\delta t_{j}$ and scattering quality factor $Q_{s_{j}}$, which is calculated from the structural parameters $a_{j}$ and $\epsilon_{j}$ (Fig. 1) using the analytical approximation for isotropic exponential media obtained by Fang and Müller (1996). The stack of layers is symmetric with respect to the free surface, which is located at the center of the stack to take into account the reflection of the wavefront.

For a given angular frequency $\omega_{c}$, the normalised coda energy envelope of a velocity seismogram at the free surface is computed from the squared amplitude envelope $A^{2}\left(t ; \omega_{c}\right)$ and is related to the energy balance within the different layers in the model through

$$
\sqrt{\frac{A^{2}\left(t ; \omega_{c}\right)}{I_{D}}}=\sqrt{\frac{2 E_{C_{N}}\left(t ; \omega_{c}\right)}{t_{N} E_{D}\left(t_{N} ; \omega_{c}\right)}}
$$

with $E_{C_{N}}\left(t ; \omega_{c}\right)$ being the spectral coda energy density of the layer containing the free surface, $t_{N}$ the traveltime from the bottom of the stack of layers to the free surface and $E_{D}\left(t ; \omega_{c}\right)$ the energy density of the direct wave at the free surface. $Q_{s}$ and $Q_{i}$ control the decay of the direct wave energy over time due to scattering and 
intrinsic attenuation via

$$
E_{D}\left(t_{j} ; \omega\right)=E_{D}\left(t_{j-1} ; \omega_{c}\right) e^{-\omega\left(t_{j}-t_{j-1}\right)\left(Q_{s_{j}}^{-1}+Q_{i_{j}}^{-1}\right)},
$$

where $t_{j}$ represents the one-way travel time through each layer. The energy balance within layer $j(j=1, \ldots, N)$ is represented by

$$
\begin{aligned}
\frac{d E_{C_{j}}}{d t}= & -\frac{1}{4 \delta t_{j}} E_{C_{j}}(t) H\left(t-t_{j}\right) \\
& -\frac{1}{4 \delta t_{j}} E_{C_{j}}(t) H\left(t-t_{j-1}\right) \\
& +\frac{1}{4 \delta t_{j-1}} E_{C_{j-1}}(t) H\left(t-t_{j-1}\right) \\
& +\frac{1}{4 \delta t_{j+1}} E_{C_{j+1}}(t) H\left(t-t_{j}\right), \\
& -\frac{\omega}{Q_{i_{j}}} E_{C_{j}}(t) H\left(t-t_{j-1}\right) \\
& +\frac{\omega}{Q_{s_{j}}} E_{D}(t) H\left(t-t_{j-1}\right) H\left(t_{j}-t\right)
\end{aligned}
$$

where $\mathrm{H}$ is the Heaviside function. The first two terms of Eq. 7 describe the energy flux from layer $j$ to the layers above and below, while the next two terms describe the opposite flux from the neighbouring layers into layer $j$. The last two terms represent the anelastic or intrinsic energy loss and the direct wave energy input into the layer. In practice, for a given model $\mathbf{m}$, comprising a single value of $a$ and $\epsilon$ for each layer in the stack, $E_{D}$ is calculated for each time sample using Eq. 6, starting from the measured energy value at the free surface. Then, the system of linear differential equations in Eq. 7 is solved for each layer in the model. Finally, synthetic coda envelopes are calculated for each frequency band using Eq. 5. 


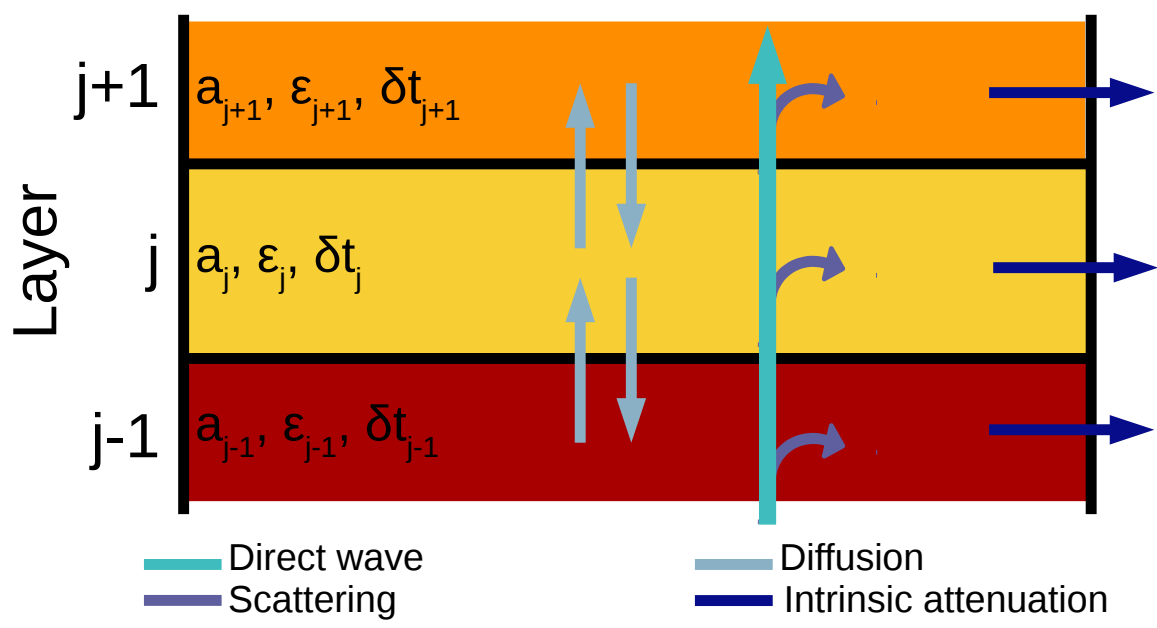

Figure 1: Total energy balance for layer $j$, according to the EFMD. (After Korn, 1997).

\subsubsection{Bayesian inference}

We use a Bayesian approach to obtain the values of the structural parameters for each layer in the model (e.g. Tarantola, 2005). In this approach, the aim is not to obtain a best fitting model, but to test a large number of models with parameters drawn from a prior probability distribution $p(\mathbf{m})$ (or prior) defined by our previous knowledge on them. In our case, we assume we have no previous knowledge on the value of the parameters and use a uniform prior.

The likelihood associated with model $\mathbf{m}, p(\mathbf{d} \mid \mathbf{m})$, is the probability of observing our data, $\mathbf{d}$, given the model parameters in $\mathbf{m}$. We used the Mahalanobis distance $\Phi(\mathbf{m})$ (Mahalanobis, 1936) between $\mathbf{d}$, with variance-covariance matrix $\mathbf{C}$, and the synthetic envelopes $g(\mathbf{m})$, to calculate the fit to our data:

$$
\Phi(\mathbf{m})=(g(\mathbf{m})-\mathbf{d})^{T} \mathbf{C}^{-1}(g(\mathbf{m})-\mathbf{d}),
$$


which we then applied to the calculation of the likelihood of model $\mathbf{m}$ :

$$
p(\mathbf{d} \mid \mathbf{m})=\frac{1}{\sqrt{(2 \pi)^{n}|\mathbf{C}|}} \exp \left(\frac{-\Phi(\mathbf{m})}{2}\right)
$$

Bayes' theorem (Bayes, 1763) allows us to calculate the corresponding sample of the posterior probability distribution (or posterior), that is, the probability density associated with model $\mathbf{m}$, or $p(\mathbf{m} \mid \mathbf{d})$ :

$$
p(\mathbf{m} \mid \mathbf{d}) \propto p(\mathbf{d} \mid \mathbf{m}) p(\mathbf{m})
$$

We create an initial model by selecting a random value for the correlation length and velocity fluctuations in all layers in the $\left(a_{\min }, a_{\max }\right)$ or $\left(\epsilon_{\min }, \epsilon_{\max }\right)$ intervals, with $a_{\min }=0.2 \lambda_{\min }[\mathrm{m}], a_{\max }=2 \lambda_{\max }[\mathrm{m}]\left(\lambda_{\min }\right.$ and $\lambda_{\max }$ being the minimum and maximum wavelengths in the layer, depending on signal frequency and background velocity), $\epsilon_{\min }=4.5 \cdot 10^{-3} \%$ and $\epsilon_{\max }=10 \%$. These maximum and minimum values were chosen considering the relevant range for detectable scattering while being geologically feasible (e.g Korn, 1993; Hock et al., 2004).

We then applied the Metropolis-Hastings algorithm (Metropolis and Ulam, 1949; Metropolis et al., 1953; Hastings, 1970) to sample the posterior probability distribution and generate our ensemble of solution models. This way, at every time step, this Markov Chain Monte Carlo (MCMC) algorithm generates a new model $\mathbf{m}^{\prime}$ by randomly choosing one of the parameters in the previous model $(\mathbf{m})$ and updating its value by adding a random number in the $(-\delta a, \delta a)$ or $(-\delta \epsilon, \delta \epsilon)$ interval, with $\delta a$ and $\delta \epsilon$ being the step size for correlation length and RMS velocity fluctuations respectively. In case the new value of the parameter exceeds the boundaries defined by $\left(a_{\min }, a_{\max }\right)$ or $\left(\epsilon_{\min }, \epsilon_{\max }\right)$, the distance $\Delta$ to the boundary is calculated and the new parameter value is forced to bounce back into the valid 
parameter range by the same distance $\Delta$. The algorithm then takes model $\mathbf{m}^{\prime}$ and uses Eqs. 7 and 5 to obtain the corresponding synthetic envelopes. In order to decide whether to accept or reject the new model, the algorithm uses the posterior probability exponent (Eq. 9), $\Phi(\mathbf{m}) / 2$, called here the loglikelihood, L, associated with model $\mathbf{m}$, as an estimator of the likelihood and the goodness of the fit to the data. Thus, if $L(\mathbf{m}) / L\left(\mathbf{m}^{\prime}\right) \geq 1, \mathbf{m}^{\prime}$ will be accepted. If $L(\mathbf{m}) / L\left(\mathbf{m}^{\prime}\right)<1$, however, it will only be accepted if $\exp \left(L(\mathbf{m})-L\left(\mathbf{m}^{\prime}\right)\right) \geq q, q$ being a random number between 0 and 1 . This algorithm ensures that parameter values closer to the true value have high likelihoods and are accepted more often than values further from the true value. The acceptance rate (AR) represents the percentage of times new parameter values were accepted through the Markov chain. There are several criteria defining what the value of the AR should be, most of them making assumptions about the properties of the target distributions (e.g. Brooks et al., 2011). In our case, since we do not have any a priori information about the posterior distributions, we aimed at AR values between 30-60 \%. Finally we calculate the 5- to 95- percentile range (PR) for each parameter in each layer in the model from our ensemble of accepted models.

For more detailed descriptions of Bayesian inference and MCMCs, we refer the reader to Tarantola (2005) or Brooks et al. (2011).

\subsubsection{Synthetic tests}

We tested our EFMD inversion code with five different synthetic datasets, with varying number of layers and parameter values. These models, together with a summary of our synthetic tests results, are shown in Table 2. In all of them, we used Pilbara Seismic Array (PSA, Section 3) as a test array and obtained its velocity model and Moho and lithosperic depths from the Australian Seismological 
Reference Model (AuSREM, Kennett and Salmon, 2012; Kennett et al., 2013; Salmon et al., 2013b), although our results should be applicable to all arrays. Frequency bands used are listed in Table 1.

Figures 2, 3 and 4 below, and S1 and S2 in the Supplementary Material, illustrate the results from our synthetic tests for Models 1 to 5 (Table 2). In order to test the convergence of our algorithm, we ran three independent Markov chains for each model, with a total of 3 million iterations (parameter combinations tested) for the single layer model, 9 million for the 2-layer models, and 15 million for the 3-layer model. In all of them, for each chain, we discarded the models corresponding to the burn-in phase, during which the algorithm is not efficiently sampling the posterior probability distribution and models are still affected by the random initialization of the Markov chain. In order to define the point at which the algorithm reached convergence and the burn-in phase ended, we first calculated the mean loglikelihood value in the second half of the chain (during which the algorithm is stable) and then subtracted $5 \%$ off that value. We consider the algorithm has converged the first time it accepts a model with loglikelihood $L$ equal or higher than this value. Our threshold was defined based on the observation, in test runs of the EFMD, that $L$ generally remained stable after reaching the defined threshold for the first time. $L$ provides an estimation of the goodness-of-fit of the synthetic data to our real data and takes negative values, meaning fits improve as $L$ gets closer to zero (Eq. 9). In terms of parameter values, we consider that a narrow 5-95 percentile range (PR) points to clearly determined values of the structural parameters, while wide 5-95 PRs would suggest multiple parameter values are equally likely and good at fitting our data.

For Model 1, with a single layer encompassing the entire lithosphere, all three chains reached stability and converged within 10000 iterations. Panels d-f in Fig. 
Table 2: Summary of the synthetic model layering and our synthetic tests results. For each model, we include the 5-95 percentile range (PR) and the acceptance rate (AR) for each parameter, as well as the maximum loglikelihood $(L)$ found during the inversion.

\begin{tabular}{|c|c|c|c|c|c|c|c|c|c|}
\hline \multirow{2}{*}{ Model } & \multirow{2}{*}{$\begin{array}{l}\text { Number } \\
\text { of layers }\end{array}$} & \multirow{2}{*}{$\begin{array}{c}\text { Layer } \\
\text { number }\end{array}$} & \multicolumn{2}{|c|}{ Input model } & \multicolumn{2}{|c|}{ Correlation length $(a)$} & \multicolumn{2}{|c|}{ RMS velocity fluctuations $(\epsilon)$} & \multirow{2}{*}{$\begin{array}{c}\text { Maximum } \\
L\end{array}$} \\
\hline & & & $a(\mathrm{~km})$ & $\epsilon(\%)$ & $5-95 \mathrm{PR}(\mathrm{km})$ & $\operatorname{AR}(\%)$ & $5-95 \mathrm{PR}(\%)$ & $\operatorname{AR}(\%)$ & \\
\hline 1 & 1 & 1 & 5.0 & 5.0 & $4.99-5.05$ & 23 & $4.99-5.00$ & 8 & -2.5 \\
\hline \multirow{2}{*}{2} & \multirow{2}{*}{2} & 1 & 2.0 & 5.0 & $1.7-2.4$ & \multirow{2}{*}{12} & $4.8-5.3$ & \multirow{2}{*}{47} & \multirow{2}{*}{-0.02} \\
\hline & & 2 & 3.0 & 4.0 & $2.8-3.4$ & & $3.9-4.1$ & & \\
\hline \multirow[b]{2}{*}{3} & \multirow{2}{*}{2} & 1 & 1.0 & 7.0 & $1.00-1.01$ & \multirow{2}{*}{51} & $6.95-7.02$ & \multirow{2}{*}{47} & \multirow{2}{*}{-0.03} \\
\hline & & 2 & 6.0 & 1.0 & $7-32$ & & $1.0-1.8$ & & \\
\hline \multirow{2}{*}{4} & \multirow{2}{*}{2} & 1 & 6.0 & 1.0 & $6-25$ & \multirow{2}{*}{50} & $1.0-1.8$ & \multirow{2}{*}{51} & \multirow{2}{*}{-1.3} \\
\hline & & 2 & 1.0 & 7.0 & $0.998-1.002$ & & $6.998-7.003$ & & \\
\hline \multirow{3}{*}{5} & \multirow{3}{*}{3} & 1 & 1.0 & 4.0 & $1-23$ & \multirow{3}{*}{52} & $0.1-4.7$ & \multirow{3}{*}{31} & \multirow{3}{*}{-0.02} \\
\hline & & 2 & 2.0 & 3.0 & $1-21$ & & $0.6-6.1$ & & \\
\hline & & 3 & 4.0 & 2.0 & $3-30$ & & $1.8-3.3$ & & \\
\hline
\end{tabular}

2 show our posterior probability density functions (PDFs) for each parameter, as well as the joint PDF. In both cases, the distributions are approximately Gaussian and symmetric, with the $5-95 \mathrm{PR}$ being $\sim 0.06 \mathrm{~km}$ and $\sim 0.01 \%$ wide for the correlation length and RMS velocity fluctuations respectively (Table 2), which indicated that the range of suitable values of the parameters is very well defined. The algorithm slightly overestimates the correlation length and underestimates the RMS velocity fluctuations, with the input value of the parameter being included in the 5-95 PR for the latter but not for the former (Table 2, Fig. 2). However, the difference between the central value of the PDFs and the true value of the parameter is $<0.4 \%$ for both the correlation length and the RMs velocity fluctuations. Graphs on the right hand side of Fig. 2 (panels g-n) show histograms of the synthetic envelopes for our ensemble of accepted models for all frequency bands. As frequency increases, both envelope amplitudes and width of the ensemble of synthetic envelopes increase too. However, in all cases, the highest density of envelopes, indicated by a dark brown color, is found in a very narrow line that matches the input data envelopes, not only in the time window used for the fit (shadowed area in the plots), but also outside of it.

Model 2 contains two layers, representing the crust and lithospheric mantle. 
Our three chains converged in less than 120000 iterations and remained stable for the rest of the inversion, as shown in panels a-c in Fig. 3. Panels $\mathrm{d}-\mathrm{i}$ in this figure summarise our results. In this case, the PDFs for the parameters in both layers are narrow (the 5-95 PR is $<0.7 \mathrm{~km}$ wide at most for $a$ and $<0.5 \%$ for $\epsilon$ ) and approximately centered around the input values, even if they are not Gaussian and show some local maxima. The true values of the parameters lie within the 5-95 $\mathrm{PR}$ in all cases, near the center of the joint PDFs, and the maximum difference between the input values and the absolute maxima of the PDFs is $2 \%$. Panels $j-q$ in Fig. 3 indicate fits to the synthetic data are good, since they show again that the largest concentration of synthetic envelopes for all frequencies coincides with the input data envelopes.

Models 3 and 4 have the same interface structure as model 2 (Table 2) and investigate high contrast situations in which a strong heterogeneity layer is above or below a layer containing weak heterogeneities respectively. Figs. S1 and S2 summarise our results and can be found in the Supplementary Material. In both cases, the chains reached stability within 11000 iterations. Posterior PDFs for the strongly scattering layer are approximately Gaussian and narrow for both models 3 and 4, with maxima that deviate from the input parameter values by $0.4 \%$ at most (Table 2). The weakly scattering layer, however, is poorly resolved for both models. The posterior PDFs for this layer are very similar in both cases and clearly non-Gaussian. They show multiple maxima that do not correspond to the input parameter values, which widens the 5-95 PR, especially for $a$. The RMS velocity fluctuation values seem to be constrained to the range from $0.5-$ $1.9 \%$ for both models, while the shape of the PDFs suggests any value of the correlation length would be equally acceptable, even if large values ( $>5 \mathrm{~km}$ ) are favoured. The stability of the chains, shown in panels a-c in Figs. S1 and S2, 
together with the ensemble of synthetic envelopes on panels $j-q$, indicate that all these models provide similarly good fits to the data and have similar loglikelihoods. This observation points to solutions being highly non-unique, and to the scattering parameters of the weakly heterogeneous layer not being easily recoverable for these high contrast cases.

Finally, model 5 contains three layers, with boundaries corresponding to upper and lower crust and lithospheric mantle. Our results are shown in Figs. 4 and Table 2. Chains converged in less than 130000 iterations. In all cases, PDFs are clearly non-Gaussian (panels d-l on Fig. 4) and have complex shapes, which widens the 5-95 PR and increases the range of suitable values of the parameters. The correlation length PDFs show clearly defined maxima near the true values of the parameter in all layers (the maximum distance between the maximum and the input parameter value being 0.35\%). RMS velocity fluctuations PDFs are more complex and neither of them show clear maxima near the input parameter values. Figure S3 contains the marginal PDFs for all parameters in all layers, as well as the PDF for each individual parameter. It shows a strong trade-off between parameter values in different layers of the model, especially the two crustal layers, and allows us to identify two independent sets of parameters from our results (see Section S.1 in the Supplementary Material for details). This interaction between the parameters is caused by two main factors: first, the energy balance the EFMD is based on (Eq. 7) is strongly dependent on the layering of the model, since the maximum energy that can be present within a layer at any time depends on its thickness (i.e. energy leaks out of thinner layers faster); second, correlation length values have a much smaller effect on coda amplitudes, compared with RMS velocity fluctuations, so the algorithm uses $\epsilon$ to compensate the excess or lack of energy within a layer and match data coda amplitudes. Since panels $\mathrm{m}-\mathrm{t}$ on Fig. 
4 do not show two clearly different sets of envelopes in our ensemble of synthetic envelopes, and given that the loglikelihood values remained stable throughout the three independent chains we ran for this example, we conclude that both sets of parameters we obtained from our inversion provide equally good fits to the data, even if neither of them match our input parameter values.

Overall, our results show that our Bayesian algorithm is capable of successfully fitting our data and retrieving the input parameter values for our 1-layer and 2layer models. For our 3-layer model, however, the method provides good fits to the data but fails to obtain the correct parameter values, so we cannot trust results from this model for real data inversions, since we do not know what the scattering parameters are beforehand. Our observations illustrate the usefulness of the Bayesian approach we took in this study. It provides detailed information about the parameter space and indicates whether a single set of parameters that fits our data exists or a range of models can equally match the data. Any estimation of scattering parameters in a maximum-likelihood framework would therefore have led to erroneous conclusions about the physical parameters in this system, which we have avoided. The joint PDFs highlight the complicated relationships and trade-offs between the model parameters in the different settings explored here, which had not been observed in previous studies using the EFMD. We do not observe systematic overestimation of $a$ in the EFMD, as reported by Hock et al. (2004). This observation might be related to the limited number of models tested in grid search approaches and the observed trade-offs between parameters. 

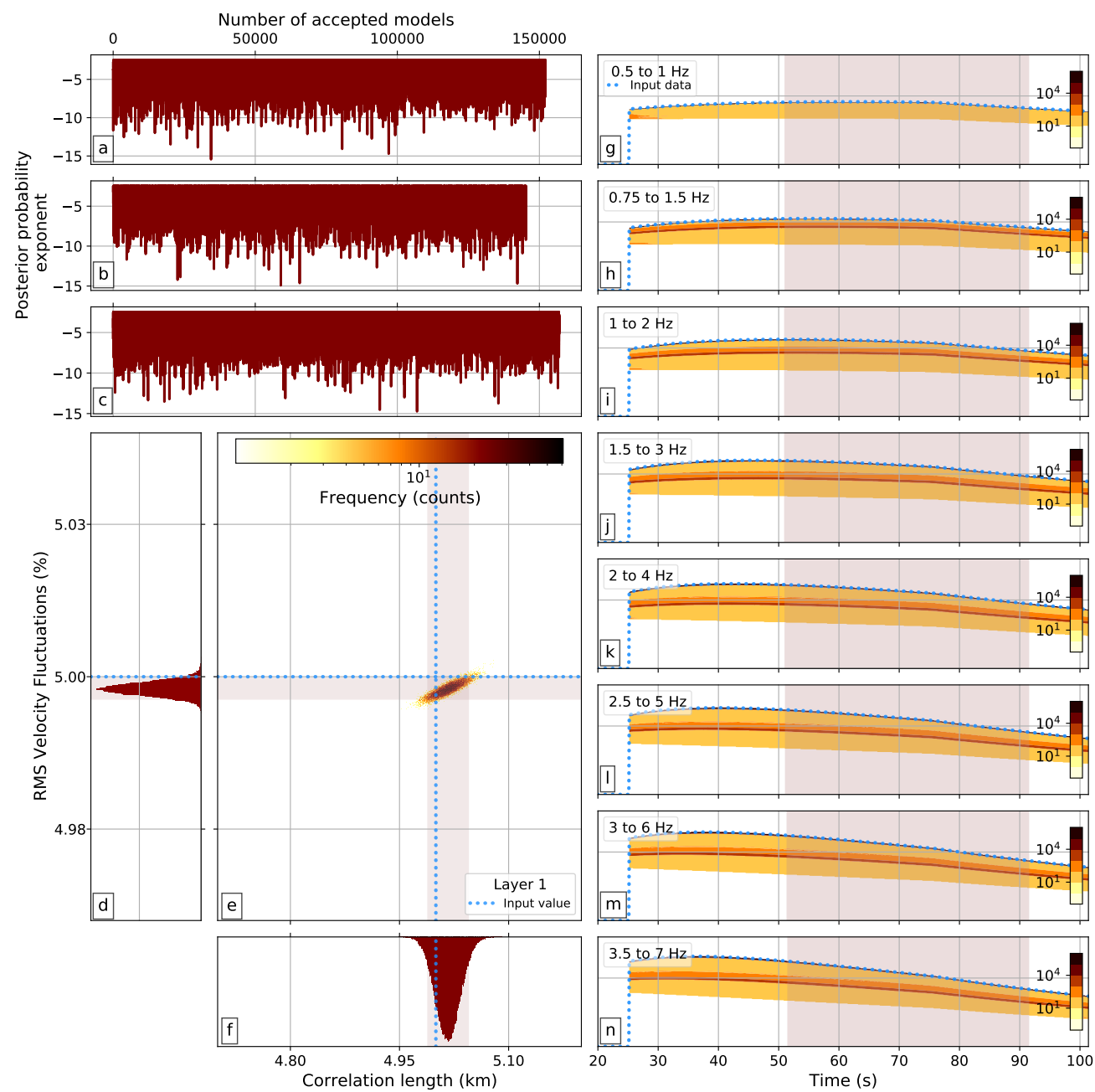

Figure 2: Summary of the results obtained from our EFMD algorithm for synthetic model 1 from Table 2 from three separate chains, adding up to a total of 3 million iterations (parameter combinations tested). Panels a-c show the loglikelihood (or posterior probability exponent) for each accepted model in the chain, once the burn-in phase was removed. Panels $\mathrm{d}-\mathrm{f}$ contain the posterior PDFs of the structural parameters, as well as the joint PDF. Dotted blue lines in these plots represent the input parameter values and the shaded area corresponds to the 5-95 percentile range (PR). Panels $\mathrm{g}-\mathrm{n}$ on the right show $2 \mathrm{D}$ histograms of the synthetic envelopes for all accepted models and frequency bands, with color bars indicating the number of models that produced a data sample within each bin. Vertical scale is the same in all plots. The shaded area here indicates the time window used for the fitting and blue dotted lines are the input data. 

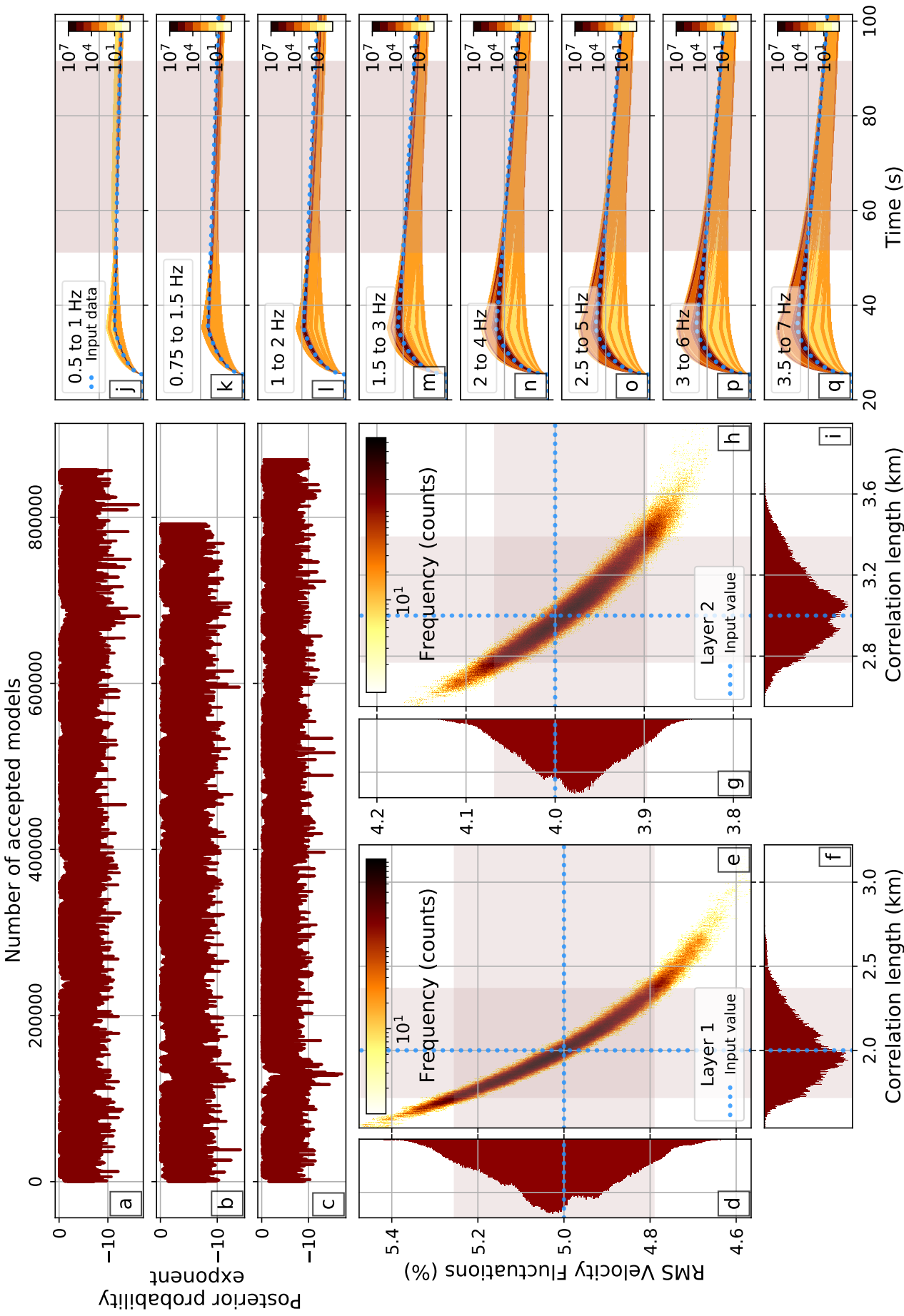

\section{咅}



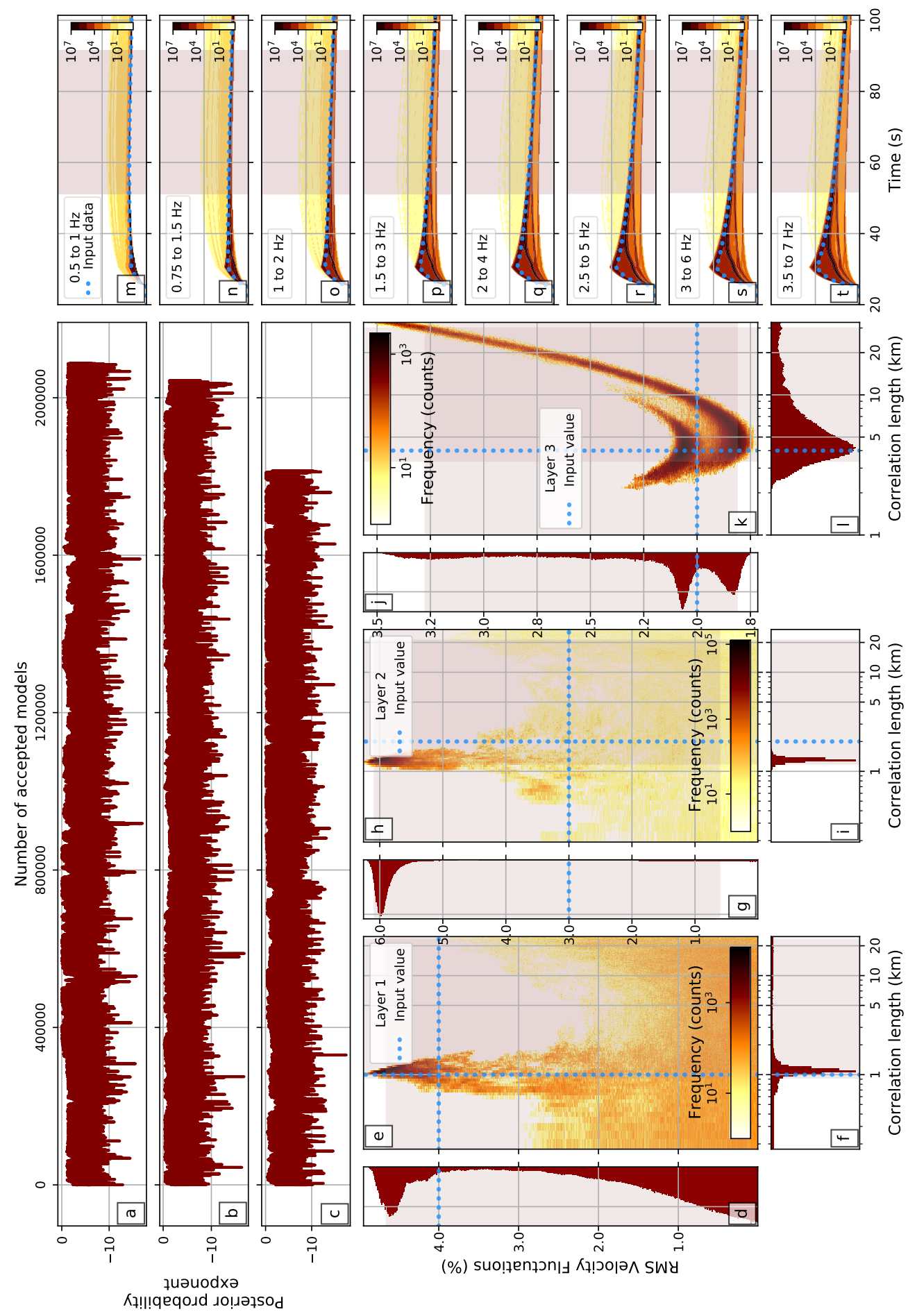

Figure 4: As Fig. 2 but for synthetic model 5 from Table 2 (3-layer model). 
Table 3: Number of events and good quality (SNR $>5$ ) traces for each array and frequency band.

\begin{tabular}{|c|c|c|c|c|c|c|c|c|c|}
\hline & \multicolumn{9}{|c|}{ Number of events per frequency band } \\
\hline & & $0.5-1 \mathrm{~Hz}$ & $0.75-1.5 \mathrm{~Hz}$ & $1-2 \mathrm{~Hz}$ & $1.5-3 \mathrm{~Hz}$ & $2-4 \mathrm{~Hz}$ & $2.5-5 \mathrm{~Hz}$ & $3-6 \mathrm{~Hz}$ & $3.5-7 \mathrm{~Hz}$ \\
\hline \multirow{2}{*}{ PSA } & Events & 86 & 161 & 213 & 276 & 343 & 268 & 212 & 158 \\
\hline & Traces & 973 & 1899 & 2489 & 3226 & 3179 & 2965 & 2282 & 1641 \\
\hline \multirow{2}{*}{ WRA } & Events & 292 & 355 & 385 & 407 & 413 & 410 & 412 & 406 \\
\hline & Traces & 709 & 843 & 916 & 977 & 983 & 984 & 980 & 965 \\
\hline ASAR & $\begin{array}{l}\text { Events } \\
\text { Traces }\end{array}$ & 309 & 375 & 440 & 429 & 405 & 397 & 386 & 374 \\
\hline
\end{tabular}

\section{DATA SELECTION AND PROCESSING}

Our dataset consists of seismic recordings from teleseismic events from January 1, 2012 to December 31, 2018, and with epicentral distances between 30 and 80 degrees from the arrays, with source depths greater than $200 \mathrm{~km}$ and magnitudes from 5 to 7 . These conditions ensure vertical or nearly vertical incidence angles and prevent near-source scattering and unwanted deep seismic phases from appearing in our time window of interest.

After removing the instrument response, we calculate the signal-to-noise ratio (SNR) for each trace and frequency band using the peak-to-peak amplitude in two separate time windows: for noise, we used a $20 \mathrm{~s}$ long window, starting $\sim 25 \mathrm{~s}$ before the theoretical P-wave arrival (as estimated from PREM (Dziewonski and Anderson, 1981)), while for the signal we chose a time window starting 1 second before the theoretical first arrival and ending 40 seconds later. Only traces with signal-to-noise ratio equal to or higher than 5 were used.

Hock et al. (2004) pointed out that the EFMD generally overestimated the RMS velocity fluctuations by up to $3 \%$ when using only vertical-component data and that a mix of 1-component and 3-component data produced unstable results, both of them caused by the difference in coda amplitudes between 1-component and 3-component data. However, the IMS arrays are dominantly vertical component, 
with WRA having three 3-component stations and ASAR a single 3-component central station. All PSA stations are three-component. To address this issue, we tried calculating a correction factor to approximate 1-component to 3-component coda levels. We used several different approaches to obtain this correction factor, all of them based on the ratio between every available 3-component coda envelope $A\left(t ; \omega_{c}\right)$ or normalised envelope (left hand side on Eq. 5) and its 1-component (vertical) counterpart. However, we found that these ratios varied significantly from event to event and frequency band to frequency band and followed complicated probability distributions, even after using our large datasets to calculate them. The corrected 1-component envelopes did not in general fully match the 3-component coda amplitudes using this approach. Our tests also showed the correction factors needed for the normalised envelopes were different than for the unnormalised ones and that small variations in coda amplitudes affected the results we got from both the EFM and EFMD. We also used the "corrected" 1-component data in our EFM-EFMD algorithm and compared the results in different settings with those from our 3-component data for PSA. In both cases, the distribution of the heterogeneity followed similar patterns, but the values of the scattering parameters and the posterior PDFs differred. Therefore, we only analyse 3-component data in this study. Table 3 shows the number of events and traces used for each array and frequency band. For PSA, we only kept events with 5 or more good quality 3component traces. For WRA and ASAR, we used all available 3-component data. This allowed us to test this method with different station configurations, from a full array (PSA) to a small group of stations (WRA) or even a single station (ASAR). In all cases, our large event dataset guarantees a thorough sampling of the structure beneath the stations and allows us to obtain robust results.

For each array, the data processing prior to the EFM/EFMD analysis was 
carried out as follows:

(i) Computation of 3-component envelopes for each frequency band, station and event. All traces were trimmed to the time window going from $t_{N}$ seconds before to $3 t_{N}$ seconds after the theoretical $\mathrm{P}$ wave arrival $\left(t_{N}\right.$ being the travel time through the lithosphere, $\sim 25 \mathrm{~s}$ for all arrays). These were then stacked by event, normalised using Eq. 5 and stacked by frequency band. Unnormalised envelopes for all events were also stacked by event and frequency band. The variance of both normalised and unnormalised envelopes was calculated sample by sample from all individual event stacked envelopes and used as the uncertainty of our data.

(ii) Estimation of $Q_{s}, Q_{i}, Q_{\text {diff }}, a$ and $\epsilon$ for a single scattering layer using the EFM.

(iii) Bayesian inversion for the structural parameters of each layer in each model type from Fig. 5 by applying the envelope modelling technique from EFMD, as described in Section 2.2, and using the $Q_{i}$ values obtained from the single layer EFM. In order to speed up this process, our data were resampled to a common sampling rate of $10 \mathrm{~Hz}$ (original sampling rates were $40 \mathrm{~Hz}$ for PSA and WRA and $20 \mathrm{~Hz}$ for ASAR) before applying the EFMD algorithm.

Background lithospheric P-wave velocities for each seismic array were obtained from the Australian Seismological Reference Model (AuSREM; Kennett and Salmon (e.g., 2012); Salmon et al. (e.g., 2013b); Kennett et al. (e.g., 2013); Salmon et al. (e.g., 2013a) (Fig. 5). 


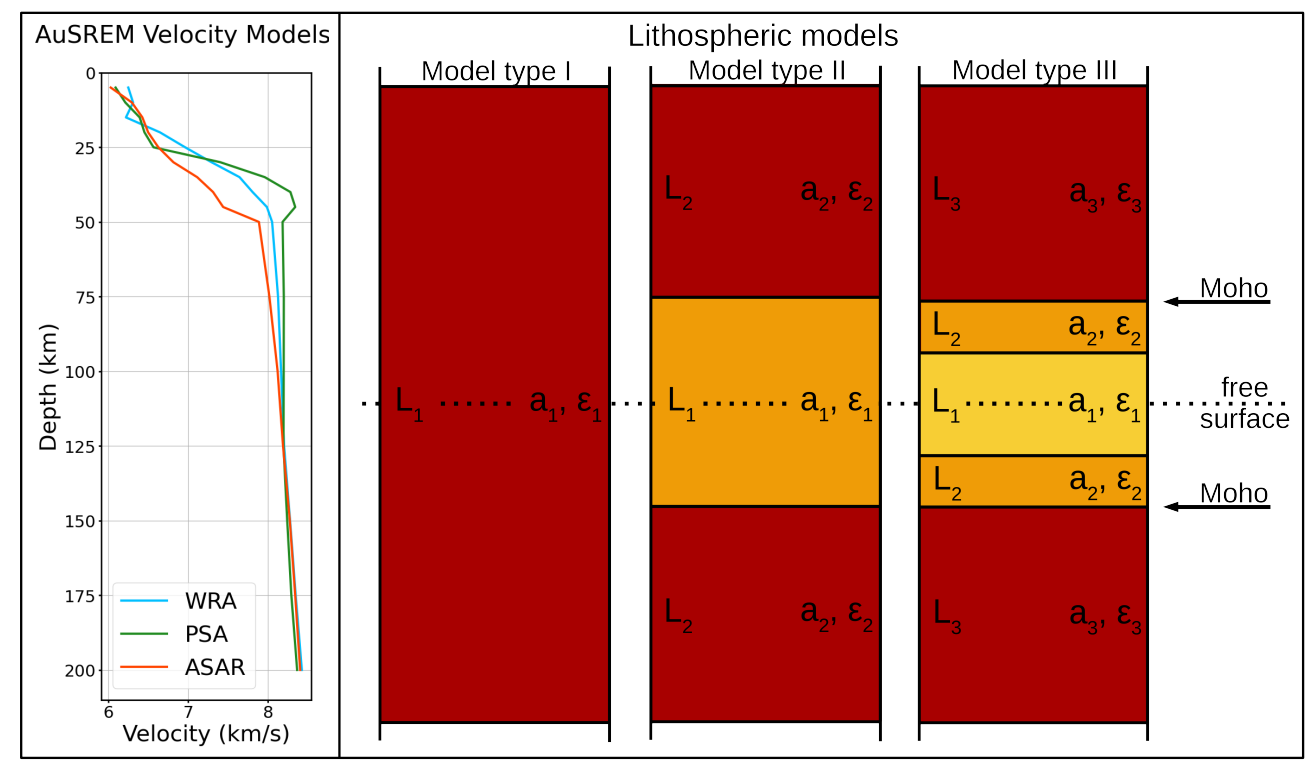

Figure 5: Representation of the AuSREM P-wave velocity models for each seismic array (left) and the three types of lithospheric models used in the EFMD (right). The layering is the same we used in the models for our synthetic tests, with Model types I, II and III corresponding to Models 1, 2 and 5 from Table 2 (Models 2, 3 and 4 have the same layering). 


\section{TECTONIC SETTING}

ASAR and WRA are located on the North Australian Craton (NAC), one of the Proterozoic cratons in the Precambrian westernmost two-thirds of the Australian continent (e.g. Myers, 1990; Simons et al., 1999; Cawood and Korsch, 2008; Wellman, 1998) (Fig. 6). The NAC consists of late Archaean to Proterozoic cratonic blocks overlaid by Proterozoic and Phanerozoic orogenic belts and basins. PSA is located on Archaean lithosphere part of the West Australian Craton (WAC), which includes both the Pilbara and Yilgarn Archaean cratons, as well as some Proterozoic orogens and basins (Cawood and Korsch, 2008) (Fig. 6). Present day tectonic activity in Australia is concentrated along the active plate boundaries in the north and east, with continental regions presenting only moderate seismicity (Fichtner et al., 2009).

Previous studies have investigated crust and lithospheric thicknesses and structure around the three arrays studied here. Thick crust $\left(L_{c}>40 \mathrm{~km}\right)$ with a wide and smooth Moho transition has generally been found in the Proterozoic shields of Central Australia while the Archaean regions of western Australia have thinner crust $\left(L_{c}<40 \mathrm{~km}\right)$ and sharper crust-upper mantle transitions (e.g. Clitheroe et al., 2000; Sippl, 2016; Salmon et al., 2013a; Kennett et al., 2011; Kennett and Saygin, 2015). This difference in crustal thickness between Archaean and Proterozoic regions seems not to fit the trend of crustal thickness increasing with age suggested for Australia (e.g. Clitheroe et al., 2000). It has been attributed to post Archaean tectonic activity underplating material at the base of the crust in these regions, as opposed to the Archaean cratons being located at passive margins and, therefore, not being affected by more recent tectonics (e.g. Drummond and Collins, 1986).

Sippl (2016) and Kennett and Sippl (2018) imaged a series of Moho offsets 


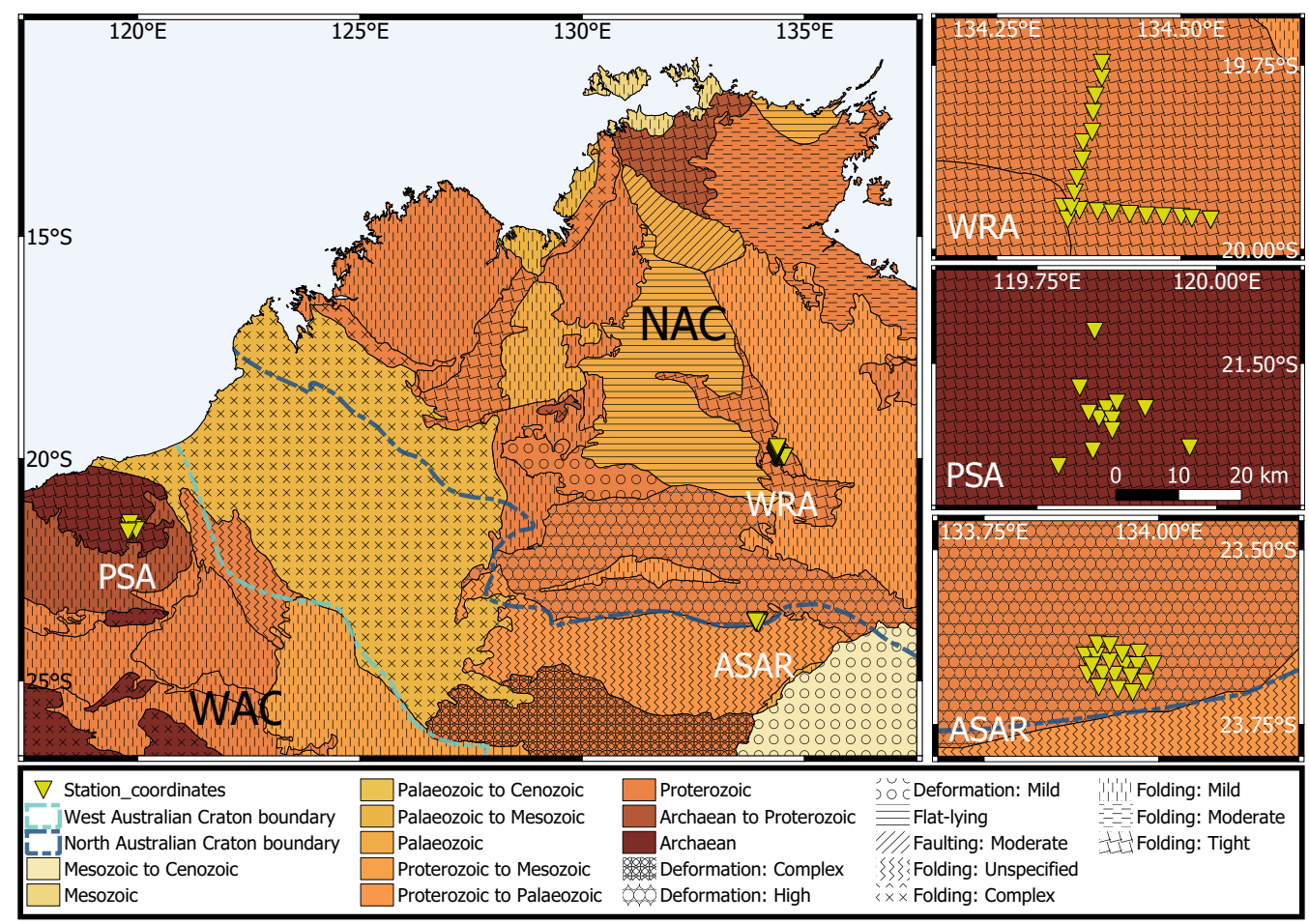

Figure 6: Simplified geological map of northwestern Australia and location of the three seismic arrays used in this study (Alice Springs Array (ASAR), Warramunga Array (WRA) and Pilbara Seismic Array (PSA)). Blue dashed lines represent the boundary of the West Australian Craton (WAC, light blue line) and the North Australian Craton (NAC, dark blue line). PSA and WRA are located on Archaean and Proterozoic basement respectively, inside the cratons, while ASAR is situated at the southern boundary of the NAC. Panels on the right show the station configuration of the arrays, with the same scale bar shown for PSA being applicable to all three maps. Geological structure based on Blake and Kilgour (1998) and Raymond et al. (2018).

along a north-south profile in the NAC. One of these offsets is associated with the Redbank Shear Zone, which separates the Aileron Province and the location of ASAR from the Amadeus Basin, just south of the array (e.g Goleby et al., 1989; Korsch et al., 1998; Sippl, 2016). The profile used in Sippl (2016) and Kennett and Sippl (2018) is located roughly $50 \mathrm{~km}$ west of ASAR and shows an offset of up to $20 \mathrm{~km}$ coinciding with ASAR latitude, even though they show constant Moho depths beneath the array. An east-west gravity anomaly has been found 
at the location of this Moho offset (Sippl, 2016, Fig. 1) and attributed to denser lithosphere at the base of the crust caused by the uplift of the Aileron crustal block during the Alice Springs Orogeny 400-350 Ma ago (Goleby et al., 1989; Aitken, 2009; Aitken et al., 2009; Sippl, 2016). This observation seems to conflict with the AusMoho model (Kennett et al., 2011, Fig. 6), which shows stable Moho depths in this part of the profile, with a slight $(\sim 2 \mathrm{~km})$ north-south depth decrease at the ASAR location. The lower resolution of the AusMoho model may be the reason for this difference. Another offset imaged by Sippl (2016) and Kennett and Sippl (2018), further north, shows a north-south decrease in Moho depth of about $10 \mathrm{~km}$ just south from WRA, which has been associated with a Proterozoic suture zone. Corbishley (1970) also found evidence of a layered and dipping structure below WRA. Gravimetric data do not show any anomalies here (Sippl, 2016), which has been attributed to a layer of sediments near the surface isostatically compensating the mass excess at depth. In this case the AusMoho model does show a sharp ( $~ 7$ $\mathrm{km})$ north-south decrease in Moho depth around this location.

Several studies have addressed the thickness of the lithosphere in the Australian continent. Some suggest similarly deep interfaces across all Precambrian cratonic regions in Australia $\left(L_{l} \approx 200 \mathrm{~km}\right.$ ) (e.g. Debayle and Kennett, 2000). More recent studies use a lithosphere-asthenosphere transition zone (LAT), defined as a mechanical or thermal boundary layer related to changes in rheology, as opposed to a simple interface at the bottom of the lithosphere (e.g. Kennett and Sippl, 2018; Yoshizawa and Kennett, 2015). Specifically, Kennett and Sippl (2018) place the upper and lower bounds of the LAT at 140 and $170 \mathrm{~km}$ depth respectively for ASAR, and at 120 and $160 \mathrm{~km}$ for WRA, while Yoshizawa and Kennett (2015) place them at 100 and $200 \mathrm{~km}$ depth for PSA. Some studies have also found evidence for mid-lithospheric discontinuities below both ASAR and WRA, at 90 
461 and $91 \mathrm{~km}$ respectively (e.g. Ford et al., 2010; Kennett and Saygin, 2015; Kennett 462 et al., 2017). 
Table 4: Summary of the main results obtained from the EFM for all arrays: intrinsic $\left(Q_{i 0}\right)$ and diffusion $\left(Q_{d 0}\right)$ quality factors values at $1 \mathrm{~Hz}$, intrinsic quality factor frequency dependence coefficient $(\alpha)$, correlation length $(a)$ and RMS velocity fluctuations $(\epsilon)$.

\begin{tabular}{cccccc}
\hline Array & $Q_{i 0}$ & $Q_{d 0}$ & $\alpha$ & $a(\mathrm{~km})$ & $\epsilon(\%)$ \\
\hline PSA & $2100 \pm 200$ & $500 \pm 40$ & 0.0 & $0.9 \pm 0.1$ & $2.9 \pm 0.1$ \\
\hline WRA & $2100 \pm 100$ & $400 \pm 20$ & 0.0 & $1.1 \pm 0.1$ & $4.5 \pm 0.1$ \\
\hline ASAR & $1000 \pm 100$ & $400 \pm 40$ & 0.2 & $0.9 \pm 0.2$ & $4.7 \pm 0.2$ \\
\hline
\end{tabular}

\section{RESULTS AND DISCUSSION}

\section{1 $\quad$ EFM results}

We calculated the coda decay rate, $a_{1}$, and its value at zero time, $a_{0}$, for all frequency bands and arrays as stated in Section 2.1. We applied the linear leastsquares fit of the squared stacked envelopes at the free surface (Fig. S4) to a time window starting $t_{N} \mathrm{~s}$ after the theoretical $\mathrm{P}$ wave arrival $\left(t_{N}\right.$ being the one-way traveltime through the lithosphere), since the EFM is only applicable after the direct wave has left the scattering layer (Korn, 1990; Hock and Korn, 2000). The length of this time window varied from 42.5 to $48 \mathrm{~s}$ for all arrays and frequency bands, depending on differences in $\mathrm{P}$ wave velocities and arrival times. Table 4 and Figure 7 summarise our EFM results for all arrays.

A least-squares fit using Eq. 2 then allowed us to calculate the quality factors for diffusion and anelasticity at $1 \mathrm{~Hz}$ from $a_{1}$. For all arrays, the coda decay rate for the lowest frequency band did not follow the trend defined by the other frequency bands. Including it in the least squares fit produced inconsistent results, and it was excluded from the analysis (Fig. S5). The intrinsic quality factor, $Q_{i}$, takes similar, frequency independent $(\alpha=0)$, values of $\sim 2000$ for WRA and PSA. For ASAR, our best fits to the coda decay rate (Eq. 2) correspond to $\alpha=0.2$ (Fig. S5) and $Q_{i} \sim 1000$. Diffusion quality factor values at $1 \mathrm{~Hz}$ are similar for ASAR 
and WRA $(\sim 400)$, and higher for PSA $(\sim 500)$. Since this quality factor does not depend on $\alpha$ (Eq. 16, Korn (1990)), this translates into $Q_{\text {diff }}$ following the same trend for all arrays but being higher for PSA than for WRA and ASAR.

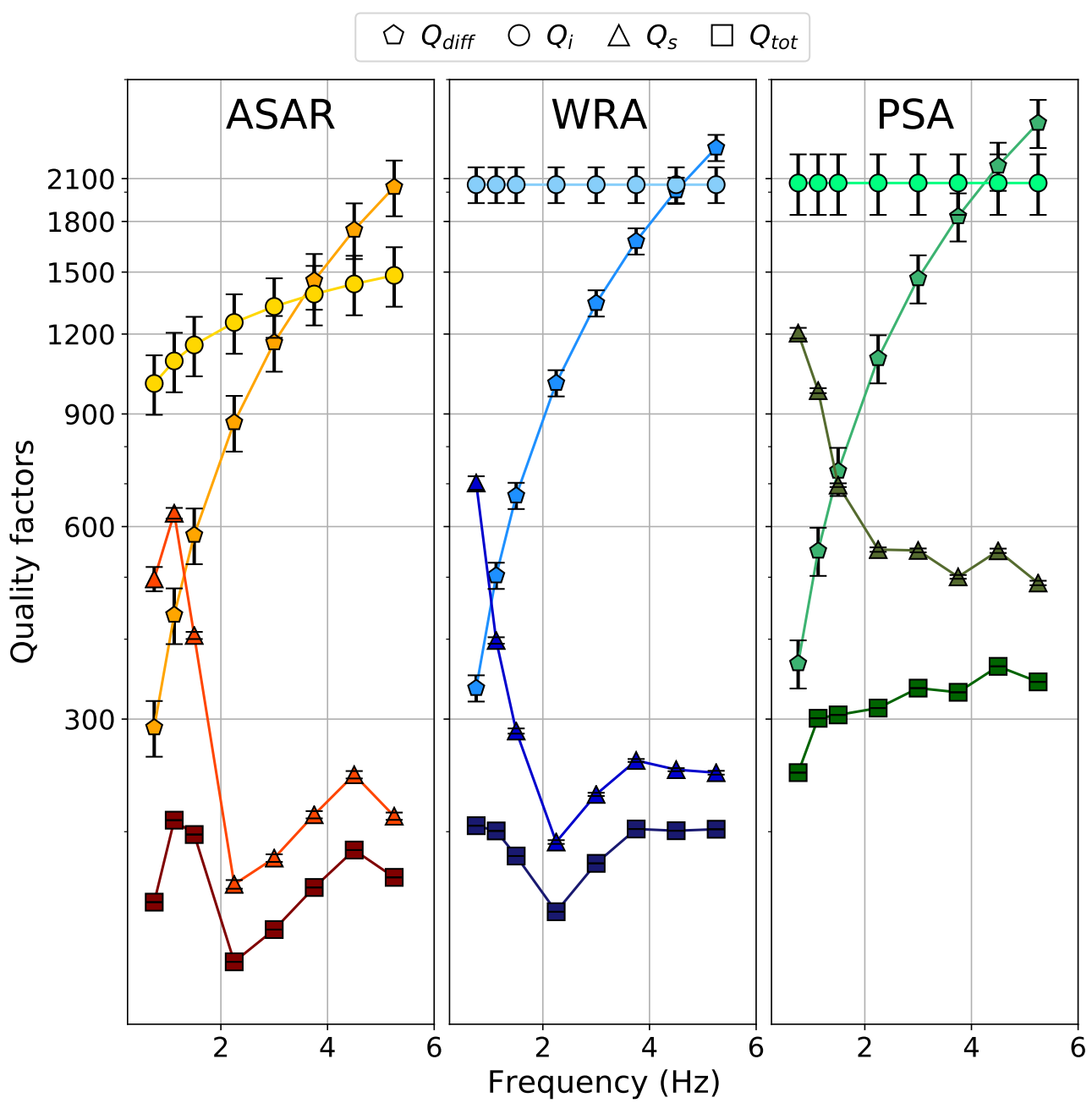

Figure 7: Frequency dependence of the intrinsic $\left(Q_{i}\right)$, the diffusion $\left(Q_{\text {diff }}\right)$, scattering $\left(Q_{s}\right)$ and total $\left(Q_{t o t}\right)$ quality factors for all arrays.

Despite the possibility to determine the type of ACF of the scattering structure using the EFM, we assumed an exponential ACF based on the similarity between different ACFs within our frequency range of interest and previous studies which 
have proposed it as an appropriate ACF for teleseismic scattering studies (Shearer and Earle, 2004). Figure S6 shows measured $Q_{s}$ values, obtained from Eq. 3, together with the theoretical least-squares regression curves derived by Fang and Müller (1996) for the relationship between the structural parameters and $Q_{s}$ for an exponential ACF. The total quality factor, $Q_{t o t}$, and $Q_{s}$ follow a similar trend. They take the highest and lowest values for PSA and ASAR respectively. For WRA and ASAR, their maximum value corresponds to the $0.5-1$ and $0.75-1.5$ $\mathrm{Hz}$ bands respectively, and the minimum for the $1.5-3 \mathrm{~Hz}$ frequency band. The frequency dependence of $Q_{s}$ and $Q_{t o t}$ for the highest frequencies is similar for both arrays. This indicates that the dominating scale length of the heterogeneity is in the $2.6-5.3 \mathrm{~km}$ range for these arrays when we consider a single scattering layer. For PSA, however, $Q_{s}$ decreases for frequencies below $1.5 \mathrm{~Hz}$ and then remains approximately constant, which could be indicative of different scale lengths of the heterogeneity being equally present in the structure. For this array, $Q_{t o t}$ increases slowly over the frequency range covered here.

In general, diffusion is the strongest attenuation mechanism (lowest Q) at low frequencies, with scattering dominating at higher frequencies. For WRA, this transition happens at $0.75 \mathrm{~Hz}$, while for ASAR and PSA, the change takes place at $1.125 \mathrm{~Hz}$. Anelasticity remains the weakest attenuation mechanism (highest Q) at low frequencies, up to $4.5 \mathrm{~Hz}$ for WRA and PSA and $3.75 \mathrm{~Hz}$ for ASAR. Above that frequency, $Q_{\text {diff }}$ becomes dominant. These results agree with the observations by Korn (1990), who obtained $Q_{i}>1000$ and $Q_{\text {diff }} \sim 300-400$ at $1 \mathrm{~Hz}$ for WRA, even if his results showed that $Q_{i}$ remained larger than $Q_{\text {diff }}$ up to $10 \mathrm{~Hz}$. Our $Q_{t o t}$ results suggest that, even if $Q_{s}, Q_{i}$ and $Q_{\text {diff }}$ are lower at most frequencies for ASAR than for the other two arrays, total attenuation strength is similar for ASAR and WRA. These lower $Q_{t o t}$ values could be related to the location of these arrays 
on the NAC, younger in origin than the WAC (Section 4). The location of ASAR, on the southern edge of the NAC, in an area widely affected by the accretionary processes that took place during the assembly of the Australian continent, as well as major events like the Petermann and Alice Springs orogens (Section 4), could explain the lower values of the different quality factors obtained for this array. For PSA, the generally high quality factors values we obtained could be related to the location of the array on a tectonically quiet Archaean craton (Section 4). Previous studies (e.g. Cormier, 1982; Korn, 1993; Sipkin and Revenaugh, 1994; Domínguez and Rebollar, 1997) have also found lower Q values in regions with quiet tectonic histories, an observation that matches our results from the EFM for all three arrays.

\subsection{EFMD results}

We used the 1-layer and 2-layer lithospheric models shown in Fig. 5 in our inversion of the data for all three arrays. $Q_{i}$ values necessary to calculate the synthetic envelopes from Eq. 5 are determined by the EFM. As with our synthetic tests, we ran three parallel Markov chains for each array and model type, with 1 million or 3 million iterations for models with 1 and 2 layers respectively. The burn-in phase, defined as described in section 2.2.2, was removed from all chains. Table 5 summarises our results. To avoid repetition, we include here only the most relevant results for each array. Figures from the rest of our inversions can be found in the Supplementary material.

Inversion of PSA data with Model type I (single layer), revealed this model produces very large amplitude codas that barely decay over time (Fig. S7). All chains were stable and converged within 14000 iterations, but the maximum loglikelihood reached during the inversion $\left(<-10^{6}\right.$, panels a-c on Fig. S7), indicated 
fits to the data are very poor, which is also obvious from the comparison of the ensemble of synthetic envelopes with the data (panels g-n on Fig. S7). The posterior PDFs suggest a nearly homogeneous lithosphere, with $\epsilon \sim 0 \%$ and $a>20 \mathrm{~km}$. This is likely due to the large thickness of the layer $(200 \mathrm{~km})$ preventing diffusion out of it and, therefore, energy levels in the heterogeneous layer remaining high at all times, regardless of the magnitude of the scattering parameters. We also tested model type I on ASAR data, since coda levels for this array are higher. These results are shown on Fig. S8. Despite the higher coda amplitudes, model type I fails to fit our data for this array, with the maximum loglikelihood reached being on the order of -10000 . ASAR coda amplitudes are similar to WRA, indicating similar behaviour. Therefore, this model was not tested for WRA.

Model type II (two layer) inversions for all three arrays showed much better fits for frequency bands D-H (Table 1) than for A-C (example for PSA in Fig. S9). However, loglikelihood values are still very low $\left(<-4 \times 10^{5}\right)$, Table 5$)$, which indicates poor fits to the data and, therefore, unreliable parameter estimations, even if there is a substantial improvement with respect to model type I. Our EFM results show scattering only becomes the dominant attenuation mechanism above $1.5 \mathrm{~Hz}$ for PSA (Fig. 7). This, together with coda amplitudes shown on panels $\mathrm{j}-\mathrm{q}$ in Fig. S9 being barely above the noise level in the time window of interest for the lowest frequency bands, suggests these codas are affected by large-scale heterogeneities and might not be composed only of energy scattered at small-scale structure. Therefore, the EFMD may not be able to fit our coda envelopes for frequencies below this threshold. To test this, we ran our EFMD inversion code for frequency bands D to $\mathrm{H}$ (Table 1) alone. By comparing our results for PSA in Fig. S9 and Fig. 8, we observe considerable improvement in the fits to the data, also evidenced by much higher loglikelihood values $(<-10)$. Given these 
Table 5: Summary of our EFMD results for all arrays and model types.

\begin{tabular}{|c|c|c|c|c|c|c|c|c|}
\hline \multirow{2}{*}{ Array } & \multirow{3}{*}{$\begin{array}{c}\begin{array}{c}\text { Model } \\
\text { type }\end{array} \\
\text { I }\end{array}$} & \multirow{2}{*}{$\begin{array}{c}\text { Frequency } \\
\text { bands }\end{array}$} & \multirow{2}{*}{$\begin{array}{c}\text { Layer } \\
\text { number }\end{array}$} & \multicolumn{2}{|c|}{ Correlation length $(a)$} & \multicolumn{2}{|c|}{ RMS velocity fluctuations $(\epsilon)$} & \multirow{2}{*}{$\begin{array}{c}\text { Maximum } \\
L\end{array}$} \\
\hline & & & & 5-95 PR (km) & AR $(\%)$ & 5-95 PR (\%) & $\mathrm{AR}(\%)$ & \\
\hline \multirow{3}{*}{$\begin{array}{c}\text { PSA } \\
3 \\
\text { comp. }\end{array}$} & & A-H & 1 & $23-32$ & 48 & $<0.01$ & 47 & $<-14 \times 10^{6}$ \\
\hline & II & A-H & $\begin{array}{l}1 \\
2\end{array}$ & $\begin{array}{l}0.5-25 \\
0.5-32\end{array}$ & 75 & $\begin{array}{l}<0.01 \\
<0.01\end{array}$ & 47 & $<-450000$ \\
\hline & II & D-H & $\begin{array}{l}1 \\
2\end{array}$ & $\begin{array}{c}0.5-0.8 \\
4-32\end{array}$ & 59 & $\begin{array}{l}2.3-2.5 \\
0.1-1.8\end{array}$ & 44 & -7.1 \\
\hline \multirow[b]{2}{*}{ ASAR } & I & $\mathrm{A}-\mathrm{H}$ & 1 & $2-30$ & 93 & $0.01-0.07$ & 44 & -10500 \\
\hline & II & D-H & $\begin{array}{l}1 \\
2\end{array}$ & $\begin{array}{c}0.2-1.4 \\
3-32\end{array}$ & 59 & $\begin{array}{l}2.4-3.0 \\
0.1-3.7\end{array}$ & 50 & -2.2 \\
\hline WRA & II & D-H & $\begin{array}{l}1 \\
2\end{array}$ & $\begin{array}{c}0.7-1.5 \\
3-32\end{array}$ & 60 & $\begin{array}{l}3.1-3.9 \\
0.2-5.0\end{array}$ & 53 & -0.7 \\
\hline
\end{tabular}

new observations, we discard frequency bands $\mathrm{A}$ to $\mathrm{C}$ (central frequencies below $1.5 \mathrm{~Hz}$, Table 1) in future inversions of the data for all arrays.

Figures 8, 9 and 10 summarise our results for all three arrays and model type II. All Markov chains converged within 10000, 7000 and 4000 iterations for PSA, ASAR and WRA, respectively. The scattering structure beneath all three arrays shows different amounts of heterogeneity in the crust and a relatively homogeneous lithospheric mantle. The posterior PDFs for both parameters in the top layer in all cases are roughly Gaussian and narrow (Table 5). Maxima for the correlation length PDFs for PSA, ASAR and WRA are at 0.6, 0.7 and $1 \mathrm{~km}$, while RMS velocity fluctuations posteriors peak at $2.4 \%, 2.7 \%$ and $3.6 \%$ respectively. PDFs for layer 2, on the other hand, show no clear maxima and also have similar shapes for all arrays. For PSA, $\epsilon$ only takes values below $\sim 3 \%$, while for WRA and ASAR, the PDF extends up to $\sim 8 \%$ and $\sim 6 \%$ respectively. In all cases, most of the accepted models have $\epsilon<1 \%$. The correlation length PDF, on the other hand, extends throughout the entire parameter space. For PSA and WRA, large values of $a(>5 \mathrm{~km})$ are favoured, while small correlation lengths $(<1 \mathrm{~km})$ seem to work better for ASAR. Loglikelihood values are high $(>-10)$ for all arrays, which suggests fits to the data are generally good. The shape of the PDFs for the bottom layer makes our solutions non-unique and similar to our results for synthetic model 
4, which had strong scattering in the crust and a fairly homogenous lithospheric mantle. This would mean scattering takes place mostly in the crust for all three arrays, with very weak or no scattering at all in the upper mantle. Finally, we used the lower and higher ends of the 5-95 PR for each scattering parameter, array and frequency band to calculate the minimum and maximum values of $Q_{s}$ in each layer. The average scattering quality factor for the lithosphere can be calculated from these values. Figure S10, in the Supplementary Material, shows a comparison between our $Q_{s}$ values from the EFM and the ones derived from the EFMD scattering parameters. In all cases, the EFM $Q_{s}$ values fall within the calculated range of values for the EFMD.

These results agree with observations from previous studies. Kennett (2015) studied P-wave reflectivity in the lithosphere and asthenosphere in Australia. Their results point to strong lithospheric heterogeneity being present beneath stations in the Proterozoic NAC and they suggest correlation lengths of at most a few kilometres and $\sim 2 \%$ velocity fluctuations in the crust. For the lithospheric mantle, they propose much larger correlation lengths $(10-20 \mathrm{~km})$ and $\epsilon<1 \%$. Kennett and Furumura (2016) and Kennett et al. (2017) also addressed the presence and interaction of multi-scale lithospheric heterogeneity in the Australian continent. In their simulations, they combined large scale heterogeneities with stochastic media and fine scale structure. Their results indicate a wide range of heterogeneity spatial scales are present and interact within the lithosphere. Their models contain four different layers for the fine scale structure, two in the crust and two in the lithospheric mantle, and different horizontal $\left(a_{H}\right)$ and vertical $\left(a_{V}\right)$ correlation lengths. Their scattering parameters suggest a mildly heterogeneous asthenospheric mantle $\left(a_{H}=10 \mathrm{~km}, a_{V}=10 \mathrm{~km}, \epsilon=0.5 \%\right)$ and an increase in the strength of the heterogeneity in the lithosphere-asthenosphere transition zone 
$\left(a_{H}=5 \mathrm{~km}, a_{V}=1 \mathrm{~km}, \epsilon=1 \%\right)$. The crust is generally more heterogeneous in these models, with $a_{H}=2.6 \mathrm{~km}, a_{V}=0.4 \mathrm{~km}$ for both crustal layers and RMS velocity fluctuations of $0.5 \%$ and $1.5 \%$ for the upper and lower crust respectively. At resolvable scales, these values are consistent with our results from the EFMD (Table 5).

\subsubsection{Limitations and assumptions}

A possible source of error in our inversion is the prescribed thickness of the layers in our models. The EFMD is sensitive to changes in the bottom depth of the different layers, especially for the shallowest layer, as this affects the diffusion out of them. For our model type II, we used a priori information on Moho and lithosphereasthenosphere boundary (LAB) depths. As discussed in Section 4, however, there is some uncertainty in reported depths, especially for the LAB. Our inversion considers the lithosphere to extend down to $200 \mathrm{~km}$ depth for all three arrays, but tests of the EFMD with shallower LABs did not produce significative changes in our results.

Other limitations of our approach are the assumptions for the determination of the different quality factors in the EFM and the fact that neither the EFM nor the EFMD take into account phase conversions and reflections at interfaces other than the free surface. Equation 15b from Korn (1990), which we use in this study, is based on the assumption that $Q_{s}$ and $Q_{\text {diff }}$ are of the same order of magnitude, even if that is not necessarily always the case. The intrinsic quality factor $\left(Q_{i}\right)$ value used in the EFMD was determined by the EFM, with a limitation to a single scattering layer and a poorly constrained frequency dependence of $Q_{i}$, since $\alpha$ could not be fully inverted for in the EFM (Section 2.1). Therefore, all layers in our EFMD models have the same $Q_{i}$ and frequency dependence as obtained in the 

and Kennett et al. (2017) could be included in future approaches of Bayesian inversion for heterogeneity structure but given the range of acceptable models we find and the trade-offs inherent in inverting for scattering parameters we have demonstrated, we are unsure if anisotropy in scattering could be well resolved with 640 these kinds of data. 

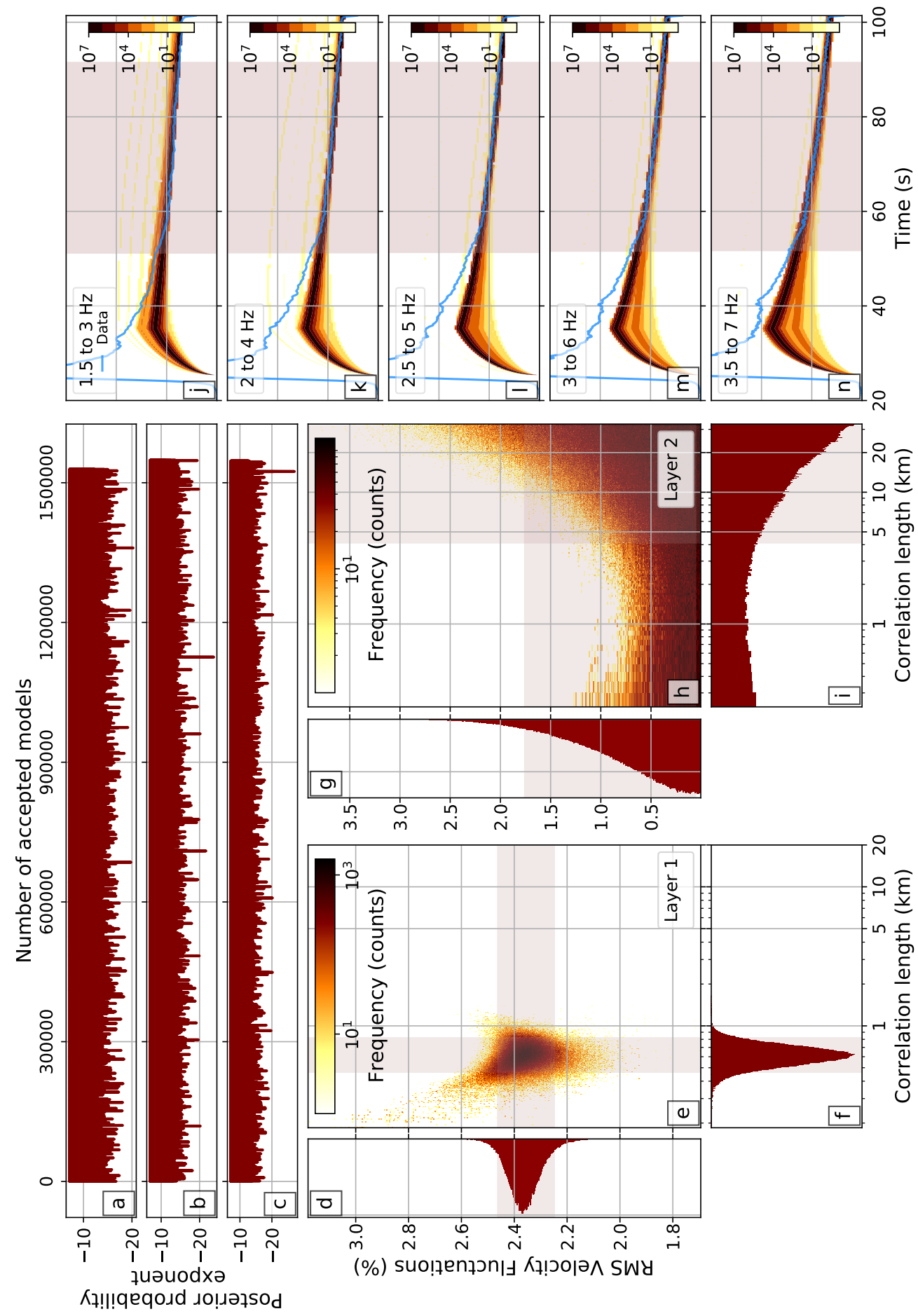

Figure 8: Results from Model type II and PSA using only the five highest frequency bands from Table 1. 


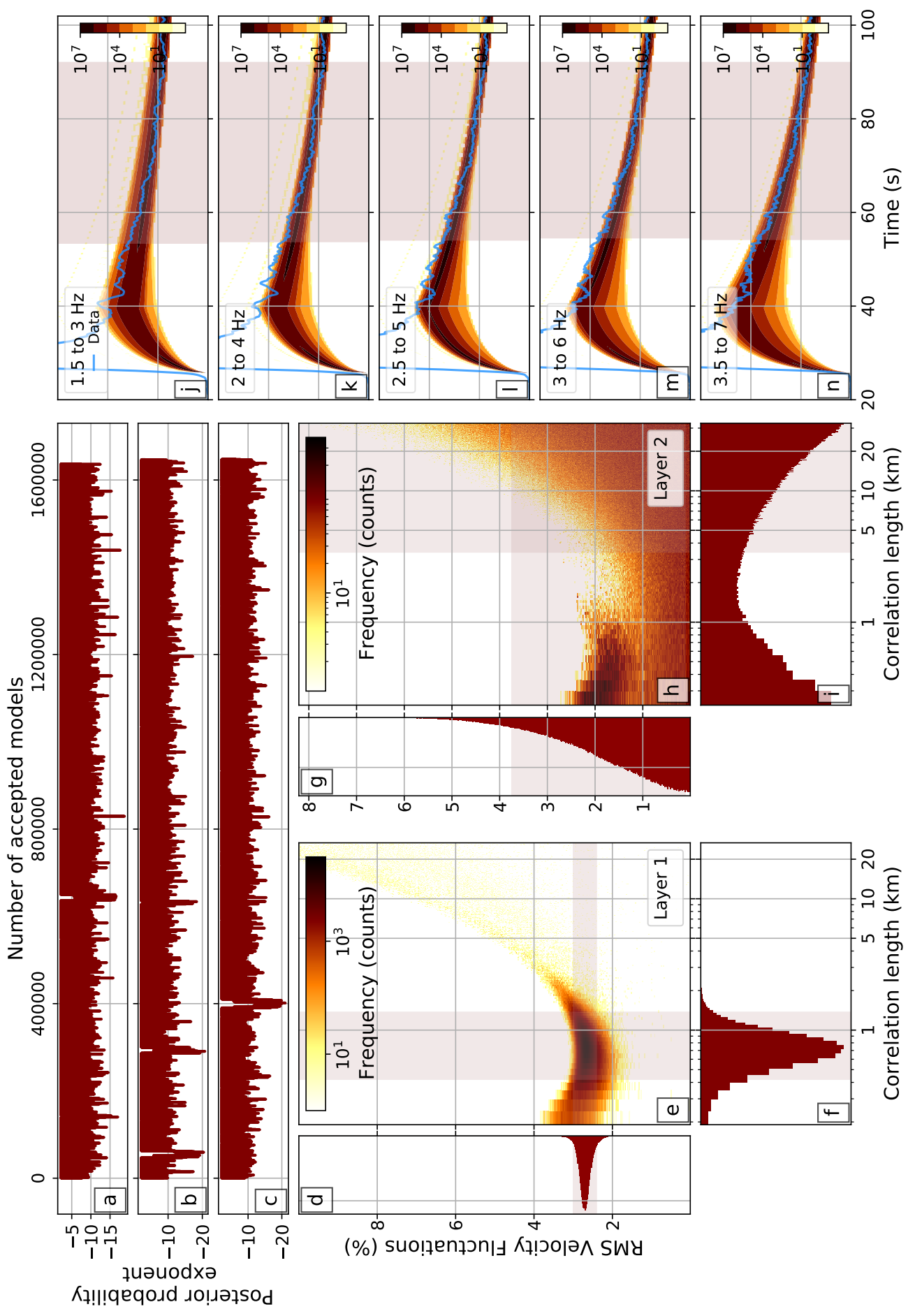

Figure 9: As Fig. 8 but for ASAR. 

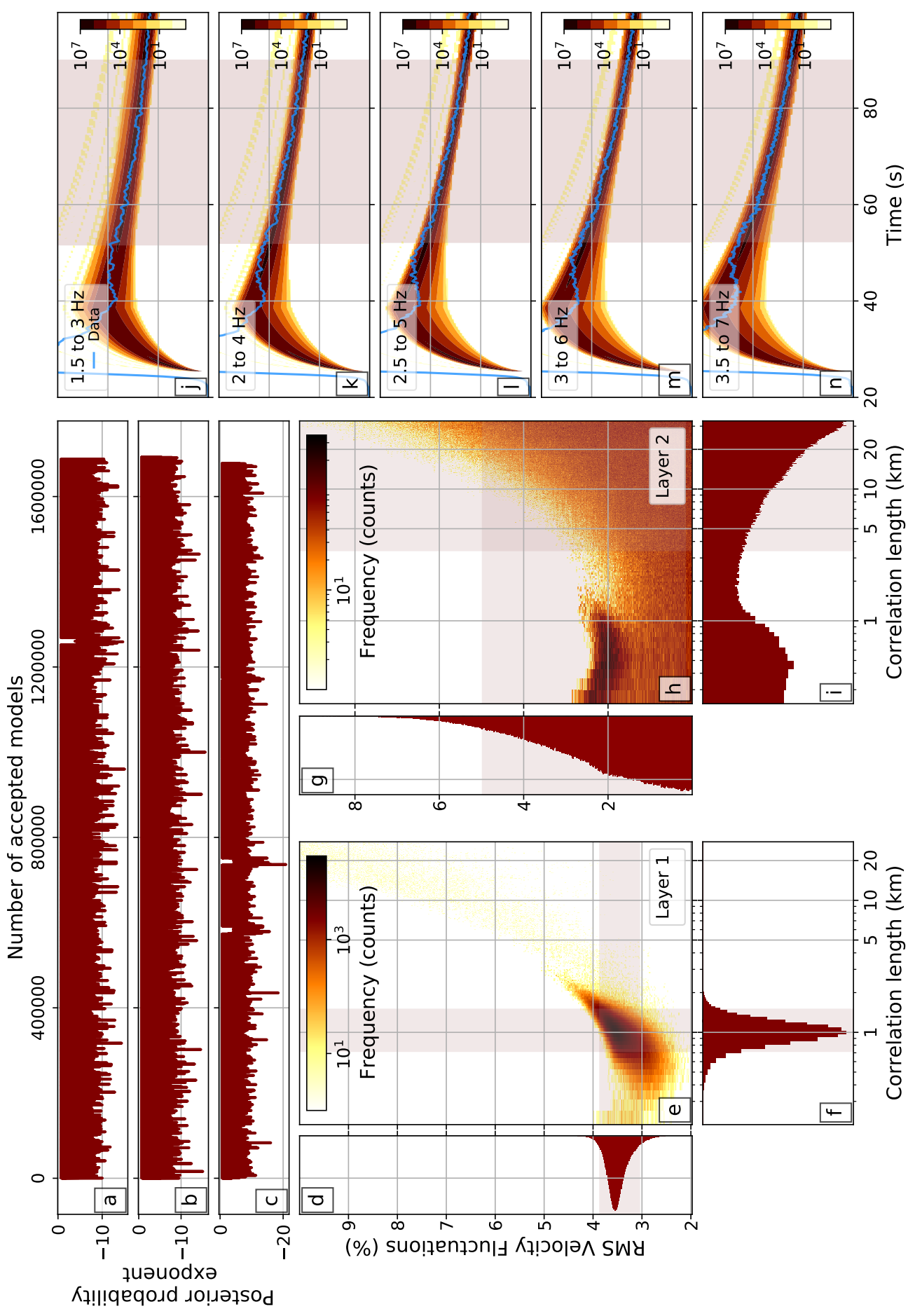

Figure 10: As Fig. 8 but for WRA. 
641

\section{CONCLUSIONS}

For three Australian seismic arrays, we applied the single layer modified Energy Flux Model (EFM) and depth dependent Energy Flux Model (EFMD) to a large dataset which includes events from a wide range of magnitudes, distances and azimuths. This ensures we are thoroughly sampling the structure of the lithosphere beneath the arrays and reduces azimuthal and lateral bias. Our EFM results highlight similarities and differences in the behaviour of the quality factors $\left(Q_{i}, Q_{\text {diff }}, Q_{s}, Q_{t o t}\right)$ for the three arrays studied here and, therefore, the attenuation structure beneath them. Generally, intrinsic and diffusion quality factors are lower at all frequencies for ASAR than for the other two arrays, which would indicate that attenuation caused by these two mechanisms would be strongest for this array. However, the scattering and total quality factors take similar values for ASAR and WRA, making their heterogeneity and overall attenuation structure comparable and different to PSA. These results are consistent with the tectonic histories and settings of the areas the arrays are located on. WRA and ASAR lie on the proterozoic North Australian Craton (NAC), but while WRA is situated near its center, ASAR is on its southern border, a margin with more complex and recent tectonic history than the interior of the craton, which correlates with the generally lower quality factor values we observe for ASAR. The EFMD confirms some of these similarities and differences. Our results suggest the crust is more heterogeneous than the lithospheric mantle for all arrays, which could be related to the cratonic nature of the lithosphere in these areas. Correlation lengths in the crust vary from $\sim 0.2-1.5 \mathrm{~km}$ and RMS velocity fluctuations take values in the 2-4\% range. The scattering structure of the lithospheric mantle, on the other hand, is more complex. Solutions for this layer are not unique, with both low $(<2 \mathrm{~km})$ and high $(>5 \mathrm{~km})$ correlation length values being equally possible. Low 
velocity fluctuation values are favoured in the inversion results for all arrays, but the posterior PDFs for ASAR and WRA extend up to $\sim 6 \%$ and $\sim 7 \%$ respectively and only to $\sim 3 \%$ for PSA, thus supporting our hypothesis that the similarities and differences in the heterogeneity structure beneath these arrays are caused by their different locations on the cratons and the different tectonic histories of these areas.

Our study highlights the suitability of Bayesian inversion approaches for the characterization of lithospheric small-scale structure. The results from our synthetic tests show that the combination of the EFMD and our Bayesian inference algorithm can effectively recover heterogeneity parameters for 1- and 2-layer models. Our approach provides detailed information about the parameter space and the trade offs and uncertainties in the determination of the structural parameters. The study of the posterior PDFs also allows us to determine whether a single set of scattering parameters can successfully explain our data or whether solutions are not unique. This ability makes the Bayesian approach to the EFMD an effective and useful tool to quantify scattering parameters in the lithosphere. 


\section{${ }_{683}$ Acknowledgments}

This work was supported by the Leeds-York Natural Environment Research Council (NERC) Doctoral Training Partnership (DTP) NE/L002574/1, in CASE partnership with the Atomic Weapons Establishment (AWE) Blacknest. Andy Nowacki was supported by NERC (standard grant REMIS: Reliable Earthquake Magnitudes for Induced Seismicity: NE/R001.154/1).

The facilities of IRIS Data Services, and specifically the IRIS Data Management Center, were used for access to waveforms, related metadata, and/or derived products used in this study. IRIS Data Services are funded through the Seismological Facilities for the Advancement of Geoscience and EarthScope (SAGE) Proposal of the National Science Foundation under Cooperative Agreement EAR-1261681.

Geoscience Australia operates the Australian National Seismic Network within Australia, its Territories and across its local region. The network supports the Joint Australian Tsunami Warning Centre, operated by Geoscience Australia and the Australian Bureau of Meteorology, which is responsible for issuing tsunami warning bulletins for Australia and its Territories. The network also contributes to monitoring earthquakes in the Australian region.

Obspy (Krischer et al., 2015) and Matplotlib (Hunter, 2007) were used for data processing and plotting. 
702

\section{References}

Aitken, A. (2009). The architecture, kinematics, and lithospheric processes of a compressional intraplate orogen occurring under Gondwana assembly: The Petermann orogeny, central Australia. Lithosphere, 1(6):343-357.

Aitken, A. R. A., Betts, P. G., Weinberg, R. F., and Gray, D. (2009). Constrained potential field modeling of the crustal architecture of the Musgrave Province in central Australia: Evidence for lithospheric strengthening due to crust-mantle boundary uplift. Journal of Geophysical Research, 114(B12):B12405.

Aki, K. (1969). Analysis of the seismic coda of local earthquakes as scattered waves. Journal of Geophysical Research, 74(2):615-631.

Aki, K. (1973). Scattering of P waves under the Montana Lasa. Journal of Geophysical Research, 78(8):1334-1346.

Aki, K. and Chouet, B. (1975). Origin of coda waves: Source, attenuation, and scattering effects. Journal of Geophysical Research, 80(23):3322-3342.

Alexander, S. S. and Phinney, R. A. (1966). A study of the core-mantle boundary using P waves diffracted by the Earth's core. Journal of Geophysical Research, 71(24):5943-5958.

Bayes, T. (1763). LII. An essay towards solving a problem in the doctrine of chances. By the late Rev. Mr. Bayes, F. R. S. communicated by Mr. Price, in a letter to John Canton, A. M. F. R. S. Philosophical Transactions of the Royal Society of London, 53:370-418.

Blake, D. and Kilgour, B. (1998). Geological Regions of Australia 
1: 5,000,000 Scale [Dataset]. Geoscience Australia, Canberra. Available at: http://pid.geoscience.gov.au/dataset/ga/32366.

Brooks, S., German, A., Jones, G., and Meng, X.-L., editors (2011). Handbook of Markov chain Monte Carlo. CRC Press.

Carpenter, E. (1966). A quantitative evaluation of teleseismic explosion records. Proceedings of the Royal Society of London. Series A. Mathematical and Physical Sciences, 290(1422):396-407.

Cawood, P. and Korsch, R. (2008). Assembling Australia: Proterozoic building of a continent. Precambrian Research, 166(1-4):1-35.

Chandrasekhar, S. (1950). Radiative Transfer. Oxford: Clarendon Press.

Christensen, N. I. and Mooney, W. D. (1995). Seismic velocity structure and composition of the continental crust: A global view. Journal of Geophysical Research: Solid Earth, 100(B6):9761-9788.

Clitheroe, G., Gudmundsson, O., and Kennett, B. L. N. (2000). The crustal thickness of Australia. Journal of Geophysical Research: Solid Earth, 105(B6):1369713713

Corbishley, D. J. (1970). Structure under Seismic Arrays. Geophysical Journal International, 21(5):415-425.

Cormier, V. F. (1982). The effect of attenuation on seismic body waves. Bulletin of the Seismological Society of America, 72(6B):S169-S200.

Debayle, E. and Kennett, B. L. N. (2000). The Australian continental upper mantle: Structure and deformation inferred from surface waves. Journal of Geophysical Research: Solid Earth, 105(B11):25423-25450. 
Domínguez, T. and Rebollar, C. J. (1997). Regional variations of seismic attenuation from coda and Lg waves in northern Baja California. Journal of Geophysical Research: Solid Earth, 102(B7):15259-15268.

Drummond, B. and Collins, C. (1986). Seismic evidence for underplating of the lower continental crust of Australia. Earth and Planetary Science Letters, 79(34):361-372.

Dziewonski, A. M. and Anderson, D. L. (1981). Preliminary reference Earth model. Physics of the Earth and Planetary Interiors, 25(4):297-356.

Etgen, J., Gray, S. H., and Zhang, Y. (2009). An overview of depth imaging in exploration geophysics. Geophysics, 74(6):WCA5-WCA17.

Fang, Y. and Müller, G. (1996). Attenuation Operators and Complex Wave Velocities for Scattering in Random Media. Pure and Applied Geophysics, 148(1):269285.

Fehler, M., Hoshiba, M., Sato, H., and Obara, K. (1992). Separation of scattering and intrinsic attenuation for the Kanto-Tokai region, Japan, using measurements of S -wave energy versus hypocentral distance. Geophysical Journal International, 108(3):787-800.

Fichtner, A., Kennett, B. L., Igel, H., and Bunge, H. P. (2009). Full seismic waveform tomography for upper-mantle structure in the Australasian region using adjoint methods. Geophysical Journal International, 179(3):1703-1725.

Flatté, S. M. and Wu, R.-S. (1988). Small-scale structure in the lithosphere and asthenosphere deduced from arrival time and amplitude fluctuations at NORSAR. Journal of Geophysical Research, 93(B6):6601. 
Ford, H. A., Fischer, K. M., Abt, D. L., Rychert, C. A., and Elkins-Tanton, L. T. (2010). The lithosphere--asthenosphere boundary and cratonic lithospheric layering beneath Australia from Sp wave imaging. Earth and Planetary Science Letters, 300(3-4):299-310.

Frankel, A. and Wennerberg, L. (1987). Energy-Flux Model of Seismic Coda: Separation of Scattering and Intrinsic Attenuation. Bulletin of the Seismological Society of America, 77(4):1223-1251.

Goleby, B. R., Shaw, R. D., Wright, C., Kennett, B. L. N., and Lambeck, K. (1989). Geophysical evidence for 'thick-skinned' crustal deformation in central Australia. Nature, 337(6205):325-330.

Hastings, W. K. (1970). Monte Carlo sampling methods using Markov chains and their applications. Biometrika, 57(1):97-109.

Helmberger, D. V. (1968). The crust-mantle transition in the Bering sea. Bulletin of the Seismological Society of America, 58(1):179-214.

Hock, S. and Korn, M. (2000). Random heterogeneity of the lithosphere across the Trans-European Suture Zone. Geophysical Journal International, 141(1):57-70.

Hock, S., Korn, M., Ritter, J. R. R., and Rothert, E. (2004). Mapping random lithospheric heterogeneities in northern and central Europe. Geophysical Journal International, 157(1):251-264.

Hunter, J. D. (2007). Matplotlib: A 2D Graphics Environment. Computing in Science $\&$ Engineering, 9(3):90-95.

Kennett, B. (2015). Lithosphere-asthenosphere P-wave reflectivity across Australia. Earth and Planetary Science Letters, 431:225-235. 
Kennett, B. and Saygin, E. (2015). The nature of the Moho in Australia from reflection profiling: A review. GeoResJ, 5:74-91.

Kennett, B. and Sippl, C. (2018). Lithospheric discontinuities in Central Australia. Tectonophysics, 744(June):10-22.

Kennett, B. L., Fichtner, A., Fishwick, S., and Yoshizawa, K. (2013). Australian seismological reference model (AuSREM): mantle component. Geophysical Journal International, 192(2):871-887.

Kennett, B. L., Yoshizawa, K., and Furumura, T. (2017). Interactions of multiscale heterogeneity in the lithosphere: Australia. Tectonophysics, 717:193-213.

Kennett, B. L. N. and Engdahl, E. R. (1991). Traveltimes for global earthquake location and phase identification. Geophysical Journal International, 105(2):429465.

Kennett, B. L. N. and Furumura, T. (2016). Multiscale seismic heterogeneity in the continental lithosphere. Geochemistry, Geophysics, Geosystems, 17(3):791-809.

Kennett, B. L. N. and Salmon, M. (2012). AuSREM: Australian Seismological Reference Model. Australian Journal of Earth Sciences, 59(8):1091-1103.

Kennett, B. L. N., Salmon, M., Saygin, E., and Group, A. W. (2011). AusMoho: the variation of Moho depth in Australia. Geophysical Journal International, 187(2):946-958.

Korn, M. (1990). A modified energy flux model for lithospheric scattering of teleseismic body waves. Geophysical Journal International, 102(1):165-175. 
Korn, M. (1993). Determination of Site-Dependent Scattering Q From P -Wave Coda Analysis With an Energy-Flux Model. Geophysical Journal International, 113(1):54-72.

Korn, M. (1997). Modelling the teleseismic P coda envelope: depth dependent scattering and deterministic structure. Physics of the Earth and Planetary Interiors, 104(1-3):23-36.

Korsch, R., Goleby, B., Leven, J., and Drummond, B. (1998). Crustal architecture of central Australia based on deep seismic reflection profiling. Tectonophysics, 288(1-4):57-69.

Krischer, L., Megies, T., Barsch, R., Beyreuther, M., Lecocq, T., Caudron, C., and Wassermann, J. (2015). ObsPy: a bridge for seismology into the scientific Python ecosystem. Computational Science \& Discovery, 8(1):014003.

Langston, C. A. (1989). Scattering of teleseismic body waves under Pasadena, California. Journal of Geophysical Research, 94(B2):1935.

Mahalanobis, P. C. (1936). On the generalized distance in statistics. National Institute of Science of India.

Margerin, L. (2005). Introduction To Radiative Transfer Theory. Geophysical Monograph-American Geophysical Union, 157:1-47.

Metropolis, N., Rosenbluth, A. W., Rosenbluth, M. N., Teller, A. H., and Teller, E. (1953). Equation of State Calculations by Fast Computing Machines. The Journal of Chemical Physics, 21(6):1087-1092.

Metropolis, N. and Ulam, S. (1949). The Monte Carlo Method. Journal of the American Statistical Association, 44(247):335-341. 
Myers, J. S. (1990). Precambrian tectonic evolution of part of Gondwana, southwestern Australia. Geology, 18(6):537.

Randall, G. E. (1994). Efficient calculation of complete differential seismograms for laterally homogeneous earth models. Geophysical Journal International, 118(1):245-254.

Rautian, T. G. and Khalturin, V. I. (1978). The use of the coda for determination of the earthquake source spectrum. Bulletin of the Seismological Society of America, 68(4):923-948.

Raymond, O., Totterdell, J., Stewart, A., and Woods, M. (2018). Australian Geological Provinces, 2018. Geoscience Australia, Canberra. Available at: http://pid.geoscience.gov.au/dataset/ga/116823.

Ritter, J. R. R., Shapiro, S. A., and Schechinger, B. (1998). Scattering parameters of the lithosphere below the Massif Central, France, from teleseismic wavefield records. Geophysical Journal International, 134(1):187-198.

Salmon, M., Kennett, B., Stern, T., and Aitken, A. (2013a). The Moho in Australia and New Zealand. Tectonophysics, 609:288-298.

Salmon, M., Kennett, B. L. N., and Saygin, E. (2013b). Australian Seismological Reference Model (AuSREM): crustal component. Geophysical Journal International, 192(1):190-206.

Sato, H. (1977). Energy propagation including scattering effects single isotropic scattering approximation. Journal of Physics of the Earth, 25(1):27-41.

Sato, H. (1984). Attenuation and envelope formation of three-component seis- 
mograms of small local earthquakes in randomly inhomogeneous lithosphere. Journal of Geophysical Research: Solid Earth, 89(B2):1221-1241.

Sato, H., Fehler, M. C., and Maeda, T. (2012). Seismic Wave Propagation and Scattering in the Heterogeneous Earth : Second Edition. Springer Berlin Heidelberg, Berlin, Heidelberg.

Shapiro, S. A. and Kneib, G. (1993). Seismic Attenuation By Scattering: Theory and Numerical Results. Geophysical Journal International, 114(2):373-391.

Shearer, P. M. and Earle, P. S. (2004). The global short-period wavefield modelled with a Monte Carlo seismic phonon method. Geophysical Journal International, 158(3):1103-1117.

Simons, F. J., Zielhuis, A., and der Hilst, R. D. (1999). The deep structure of the Australian continent inferred from surface wave tomography. Lithos, 48:17-43.

Sipkin, S. A. and Revenaugh, J. (1994). Regional variation of attenuation and travel time in china from analysis of multple-scs phases. Journal of Geophysical Research: Solid Earth, 99(B2):2687-2699.

Sippl, C. (2016). Moho geometry along a north-south passive seismic transect through Central Australia. Tectonophysics, 676:56-69.

Tarantola, A. (2005). Inverse problem theory and methods for model parameter estimation, volume 89. Siam.

Thorbecke, J., Slob, E., Brackenhoff, J., van der Neut, J., and Wapenaar, K. (2017). Implementation of the Marchenko method. Geophysics, 82(6):WB29WB45. 
van der Neut, J., Wapenaar, K., Thorbecke, J., Slob, E., and Vasconcelos, I. (2015). An illustration of adaptive Marchenko imaging. The Leading Edge, $34(7): 818-822$.

Wellman, P. (1998). Mapping of geophysical domains in the Australian continental crust using gravity and magnetic anomalies. In Structure and evolution of the Australian continent, pages 59-71. American Geophysical Union, Washington, D.C: AGU.

Wu, R.-S. (1985). Multiple scattering and energy transfer of seismic waves - separation of scattering effect from intrinsic attenuation - I. Theoretical modelling. Geophysical Journal International, 82(1):57-80.

Wu, R.-S. and Aki, K. (1988). Introduction: Seismic Wave Scattering in Threedimensionally Heterogeneous Earth. In Scattering and Attenuations of Seismic Waves, Part I, pages 1-6. Birkhäuser Basel, Basel.

Yoshizawa, K. and Kennett, B. L. N. (2015). The lithosphere-asthenosphere transition and radial anisotropy beneath the Australian continent. Geophysical Research Letters.

Zelt, C. A. and Barton, P. J. (1998). Three-dimensional seismic refraction tomography: A comparison of two methods applied to data from the Faeroe Basin. Journal of Geophysical Research: Solid Earth, 103(B4):7187-7210. 


\section{S SUPPLEMENTARY MATERIAL}

\section{S.1 Additional synthetic tests results}

Figures S1 and S2 contain the results from our synthetic tests for models 3 and 4 on Table 2. Both of them are two-layer models, so three independent Markov chains, each one 3 million iterations long, were combined to produce these figures. Figure S3 contains the marginal PDFs for all parameters in all layers, as well as the PDF for each individual parameter. These plots illustrate the presence of two independent families of parameters that separately fit our data within our posterior PDFs. Subindices $L i$ ( $i$ being the layer number) are used here to refer to parameter values in each layer of the model. Starting on panel $3-1$, we observe how all accepted models corresponding to $a_{L 1} \sim 1 \mathrm{~km}$ (the sharp peak on panel 1-1) have $a_{L 2}$ values either lower than $1 \mathrm{~km}$ or higher than $2 \mathrm{~km}$, which would correspond to the tails of the $a_{L 2} \mathrm{PDF}$ on panel 3-3. Panel 3-2 compares $\epsilon_{L 1}$ and $a_{L 2}$ and we can see how all models with $a_{L 2}$ in the ranges mentioned before have $\epsilon_{L 1}>3.5 \%$. Similarly, panels $4-1,5-2$ and 5-3 show that these values of $a_{L 1}, a_{L 2}$ and $\epsilon_{L 1}$ correspond to $\epsilon_{L 2}<\sim 5 \%, \epsilon_{L 3} \sim 2.1 \%$ (the second, sharp, peak on the PDF on panel $6-6$ ) and $2<a_{L 3}<10 \mathrm{~km}$ (the wide peak on the PDF on panel 5-5). Interestingly, the first peak and the side tail on the $\epsilon_{L 3} \operatorname{PDF}$ (panel 6-6) correspond to the same parameter family, as do the tail and the base of the peak on the $a_{L 3}$ PDF (panel 5-5). Following the same reasoning detailed above, we extracted the other family of parameters, which are summarised on Table S1. 
Table S1: Summary of the two independent and equally likely families of parameters extracted from Fig. S3 for our synthetic model 3 from Table 2.

\begin{tabular}{ccccccc}
\hline & $\begin{array}{c}a_{L 1} \\
(\mathrm{~km})\end{array}$ & $\begin{array}{c}\epsilon_{L 1} \\
(\%)\end{array}$ & $\begin{array}{c}a_{L 2} \\
(\mathrm{~km})\end{array}$ & $\begin{array}{c}\epsilon_{L 2} \\
(\%)\end{array}$ & $\begin{array}{c}a_{L 3} \\
(\mathrm{~km})\end{array}$ & $\begin{array}{c}\epsilon_{L 3} \\
(\%)\end{array}$ \\
\hline Input model & 1.0 & 4.0 & 2.0 & 3.0 & 4.0 & 2.0 \\
\hline Parameter family 1 & $\sim 1$ & $>3.5 \%$ & $<1 \&>2$ & $<5$ & $2-10$ & $\sim 2.1$ \\
\hline Parameter family 2 & $<0.6 \&>1.1$ & $<3.5$ & $\sim 1.2$ & $\sim 6$ & $3-30$ & $\sim 1.6-3.7$ \\
\hline
\end{tabular}



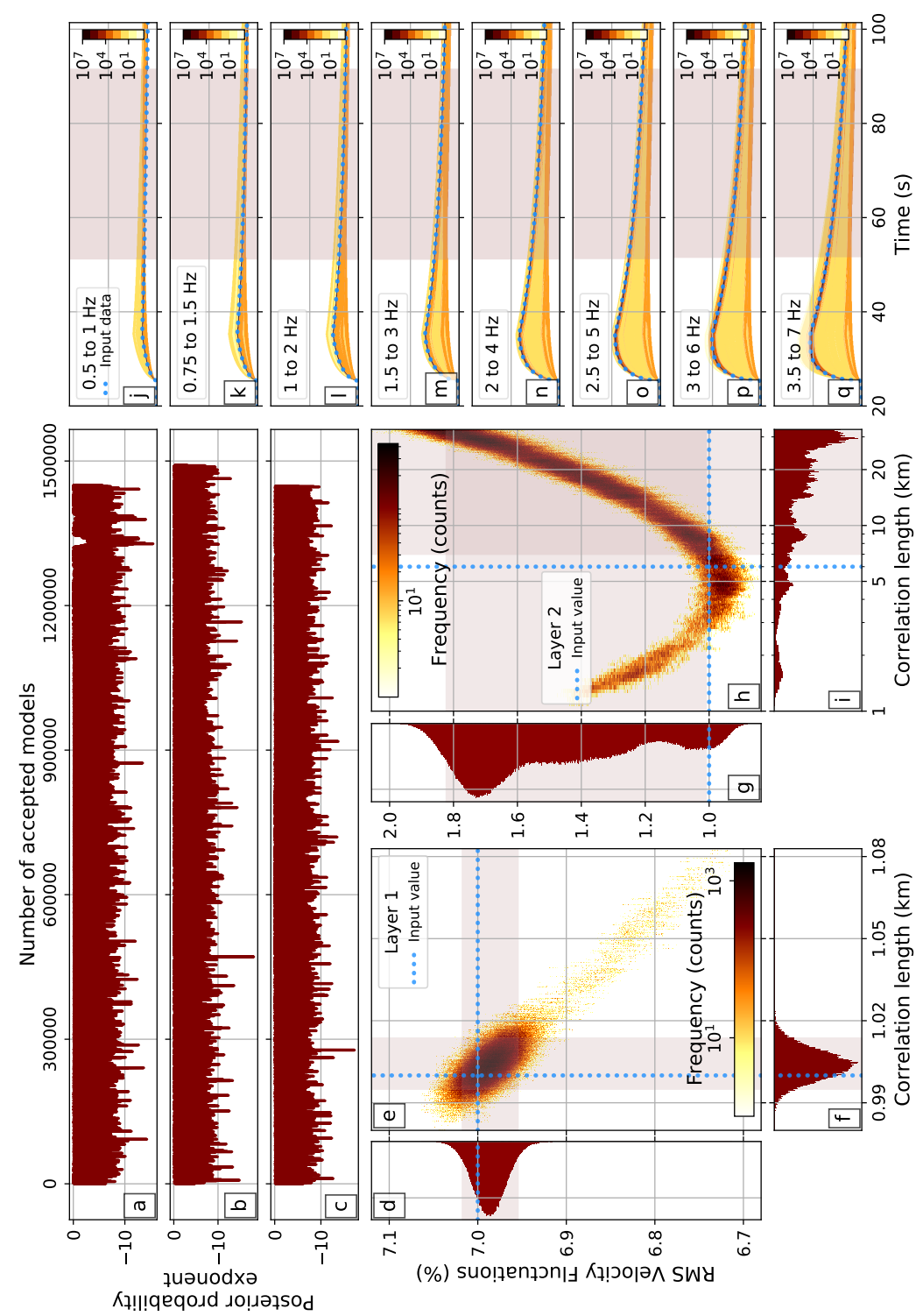

Figure S1: Results from our synthetic test of model 3 from Table 2, in which a strongly scattering layer lies above a weakly scattering one. Panels a-c show the loglikelihood for each accepted model in the chain, while $\mathrm{d}-\mathrm{i}$ contain the posterior PDFs of the structural parameters and the joint PDF. Dotted blue lines in these plots represent the input parameter values and the shaded area indicates the 5-95 percentile range (PR). Panels j-q on the right contain 2D histograms of the synthetic envelopes for all accepted models, with color bars indicating the number of models that produced a data sample within each bin of the grid. Vertical scale is the same in all plots. The shaded area in these panels points to the extent of the time window used for the fitting. 

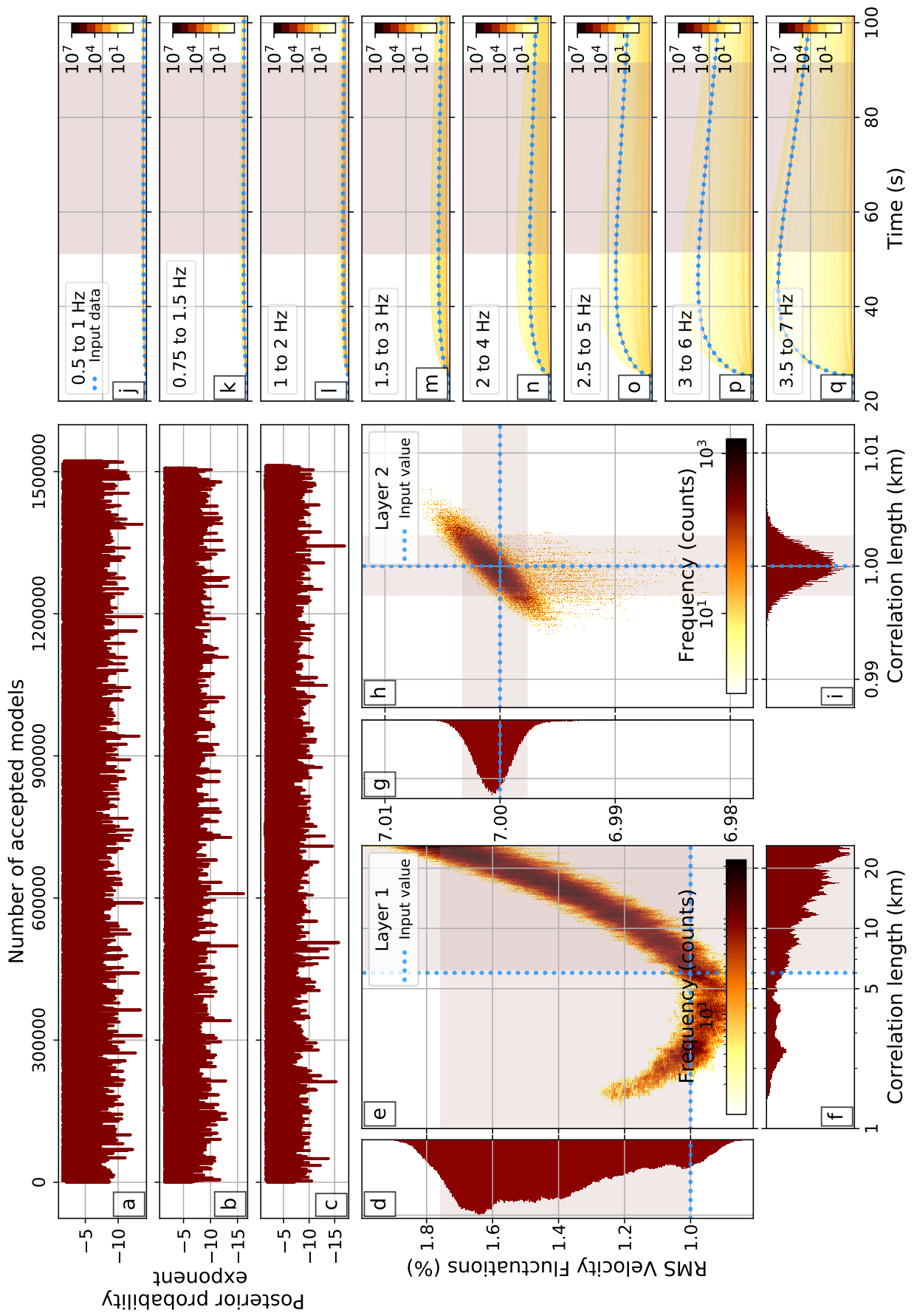

Figure S2: Results from our synthetic test of model 4. In this case, the top layer contains weak heterogeneities, while the bottom layer is highly heterogeneous. 


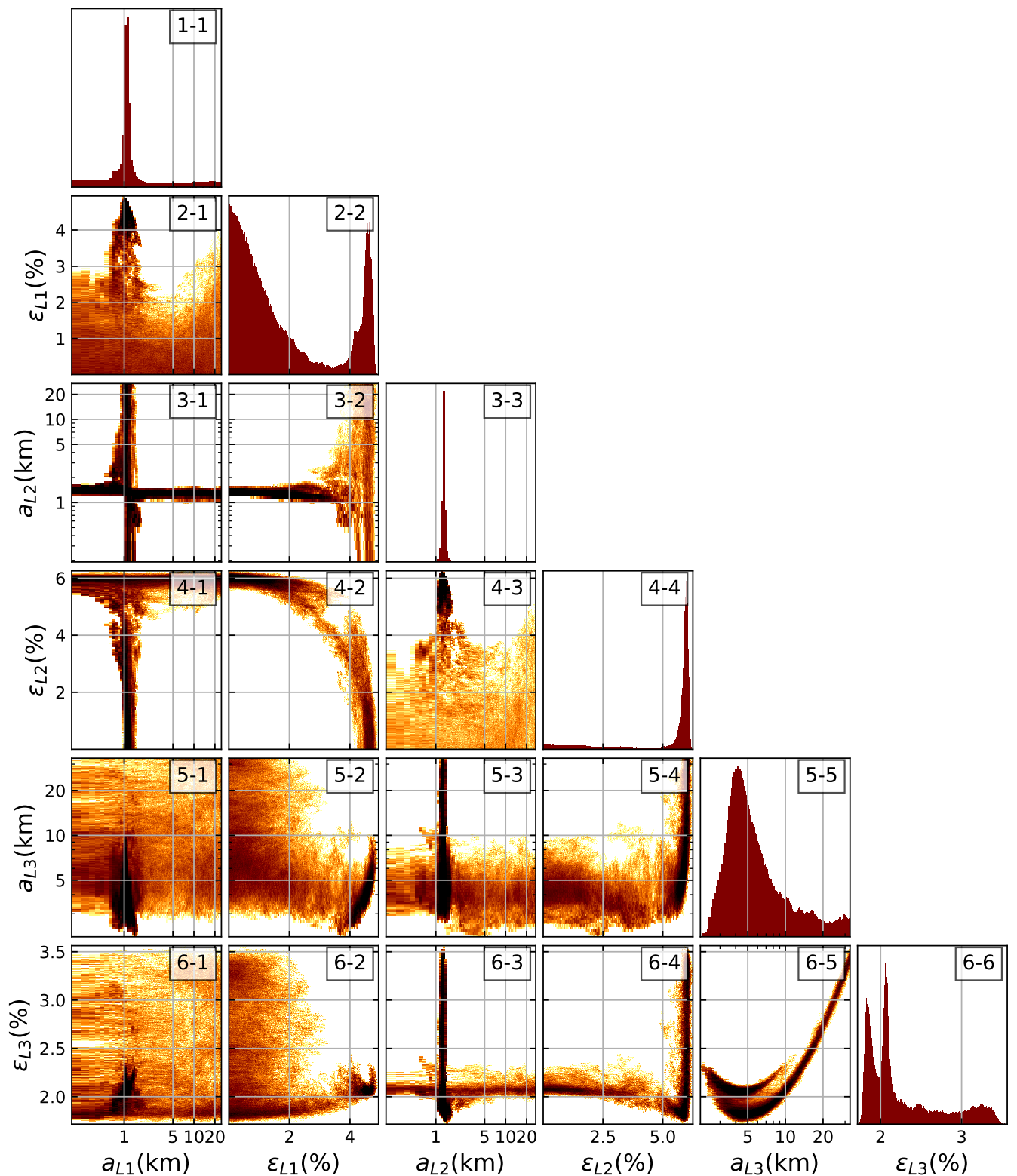

Figure S3: Joint PDFs for all parameters and layers in synthetic model 5 from Table 2. Plots in the diagonal of the figure contain the individual PDF for the different scattering parameters. 


\section{S.2 Additional EFM results}

Figures S4, S5 and S6 show our results from the different least-squares fits necessary to obtain our final EFM results, as described in Section 2.1.
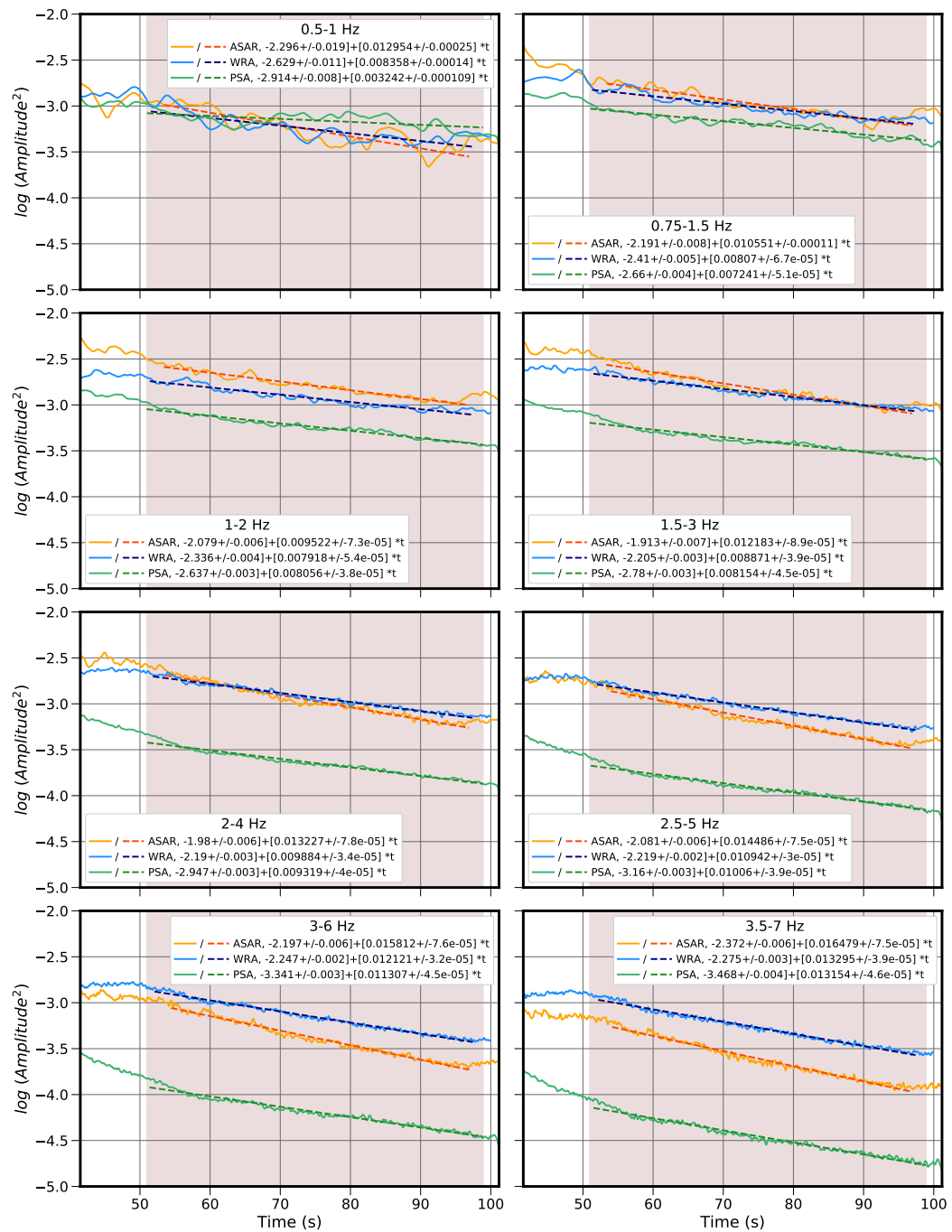

Figure S4: Linear fit of the logarithm of the squared normalised coda envelopes for all arrays, as described in Section 2.1. The shaded area represents the maximum time window used for the fits. Lighter solid lines represent our data envelopes. Darker, dashed lines show the linear fits whose equations are shown in the legend. 


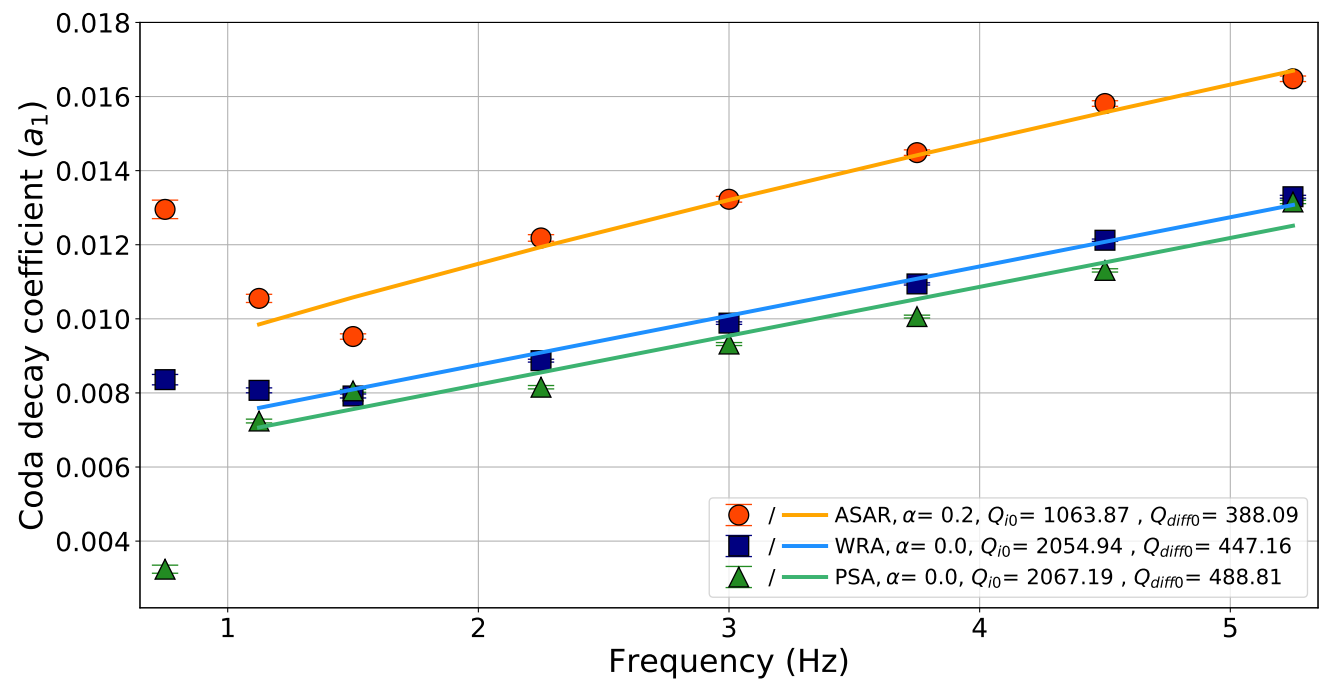

Figure S5: Coda decay coefficient $\left(a_{1}\right)$ vs. frequency for all arrays. Solid lines represent the regression curves defined by Eq. 18 from Korn (1990). The legend contains our obtained values of the intrinsic and diffusion quality factors at $1 \mathrm{~Hz}$, as well as the indicative estimation of the thickness of the scattering layer.

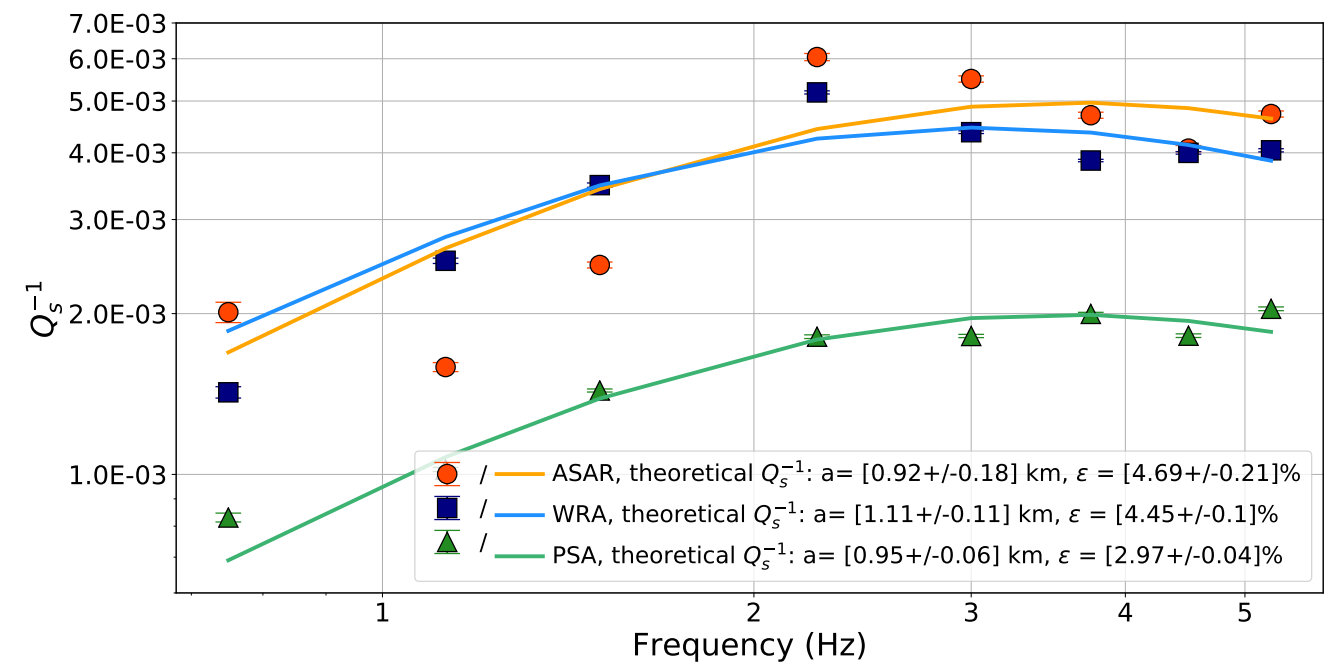

Figure S6: Scattering quality factor, Qs, vs. the theoretical curve derived by Fang and Müller (1996). The legend contains our estimation of the correlation length and RMS velocity fluctuations for a single scattering layer. 


\section{S.3 Additional EFMD results}

Results from EFMD inversions from our initial tests. In all cases, three independent Markov chains have been combined to produce these results, each one 1 or 3 million iterations long for models with 1 or 2 layers respectively. The burn in phase has been removed from all of them, as described in Section 2.2.2. Panel content in all these figures is as described in Section S.1.

We used the relationship between $Q_{s}^{-1}$ and the structural parameters derived by Fang and Müller (1996) to calculate the scattering quality factor for each array, frequency band and model layer. The $5-95$ percentile range (PR) for each parameter provides minimum and maximum $Q_{s}$ values for each layer. The equation below, based on the definition of attenuated time given by Carpenter (1966), allows us to calculate the average scattering quality factor for the lithosphere, which we can then compare with our $Q_{s}$ values from the EFM:

$$
\frac{t_{\text {total }}}{Q_{\text {saverage }}}=\frac{t_{1}}{Q_{s 1}}+\frac{t_{2}}{Q_{s 2}},
$$

where $t_{\text {total }}$ represents the total traveltime through the lithosphere and $t_{i}$ and $Q_{s i}$ $(i=1,2)$ the traveltime and scattering quality factor for each layer in the model respectively. Figure S10 shows a comparison between our EFM $Q_{s}$ values for the single-layer model and the ones calculated for the EFMD using Eq. S1. 


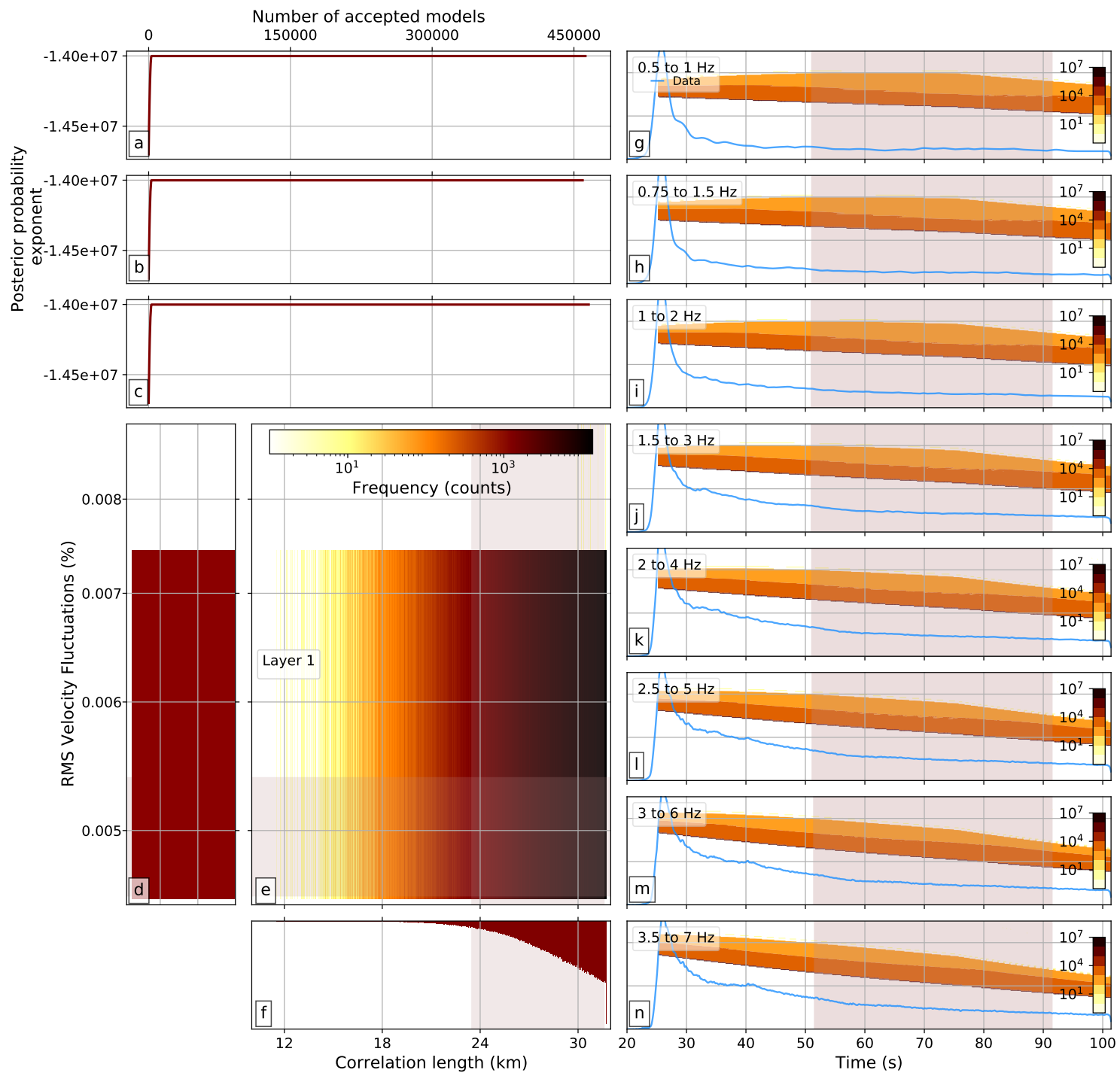

Figure S7: EFMD results for PSA and model type I. 


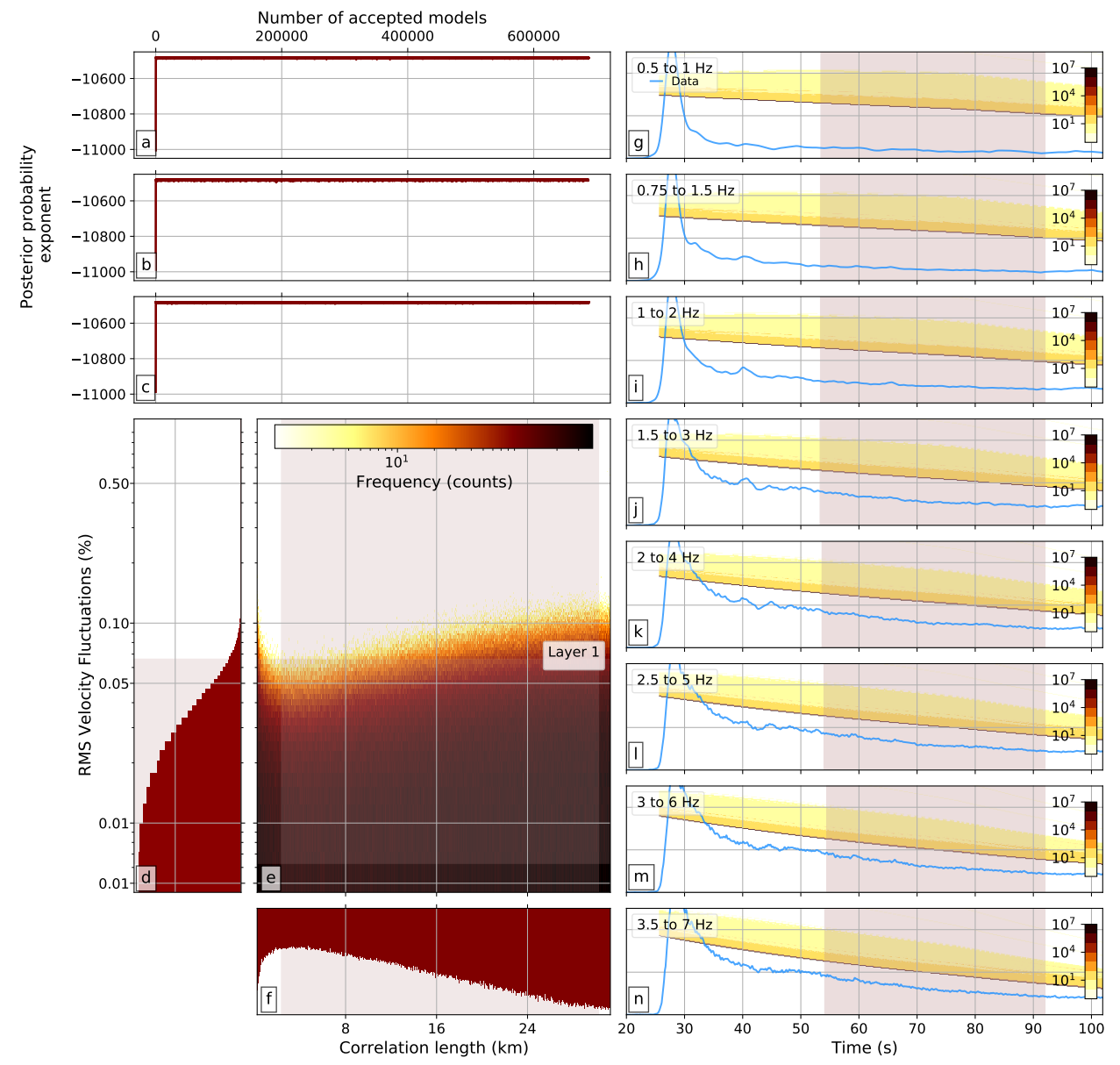

Figure S8: EFMD results for ASAR and model type I. 

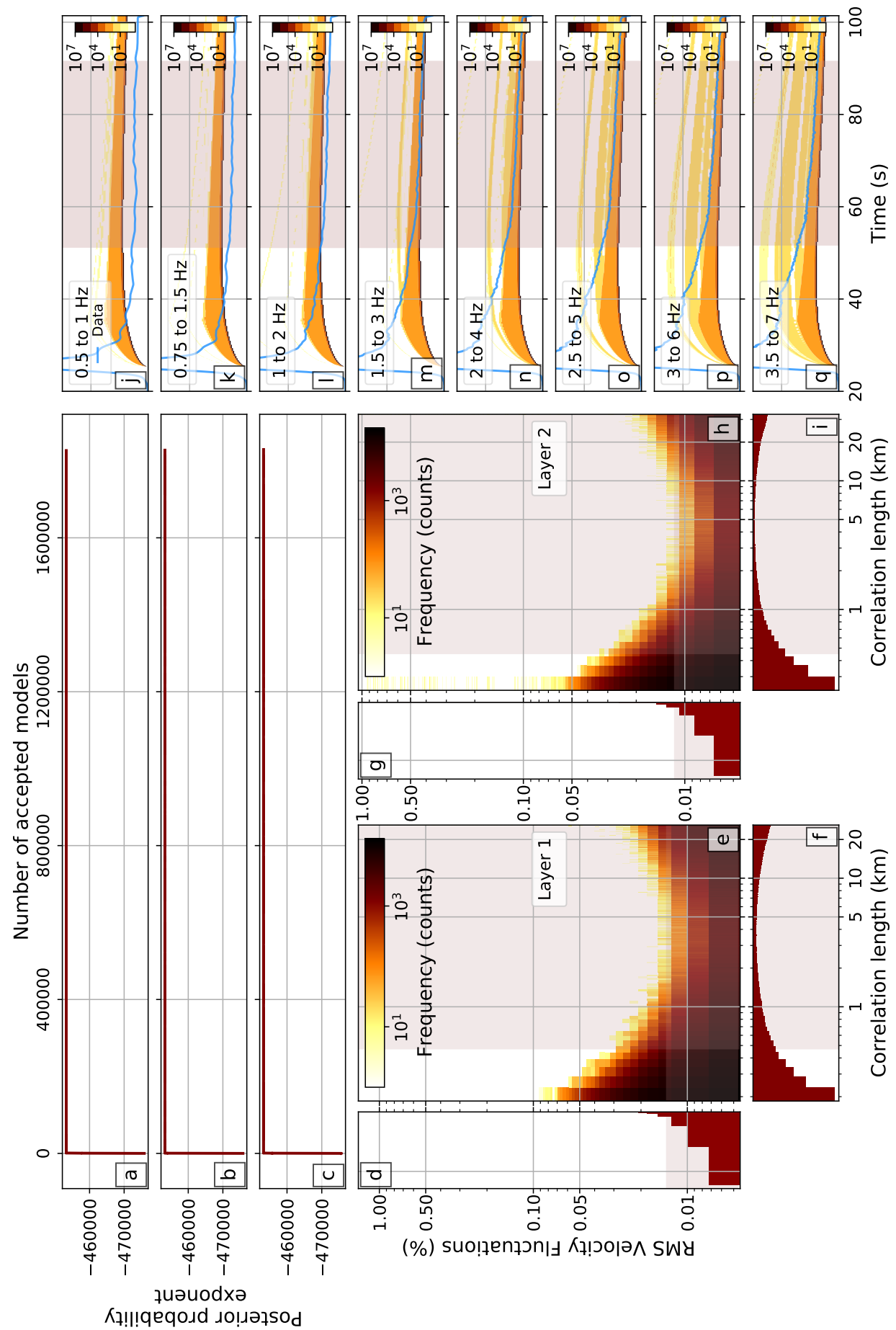

Figure S9: EFMD results for PSA and model type II using all eight frequency bands listed on Table 1. 


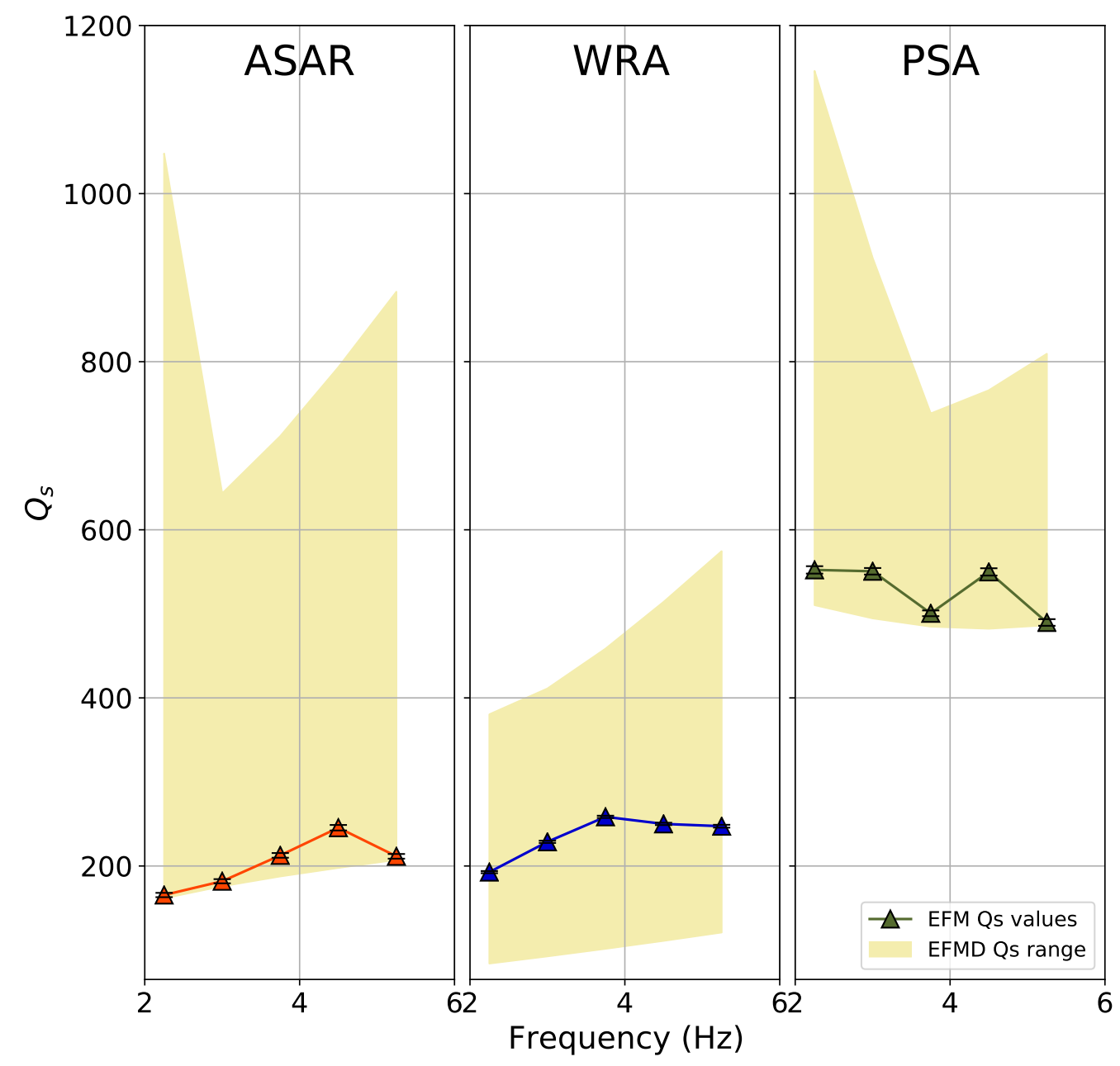

Figure S10: Comparison between the $Q_{s}$ values obtained from the EFM and calculated from the EFMD structural parameters. Shaded area represents the range between the minimum and maximum $Q_{s}$ values, derived using the lower and higher ends of the $5-95$ PR for each parameter in each layer and Eq. S1. 